\title{
Nonlinear dynamics and pattern formation in turbulent wake transition
}

\author{
By RONALD D. HENDERSON \\ Aeronautics and Applied Mathematics, \\ California Institute of Technology, Pasadena, CA 91125, USA \\ (Received 1 October 1996 and in revised form 17 July 1997)
}

\begin{abstract}
Results are reported on direct numerical simulations of transition from twodimensional to three-dimensional states due to secondary instability in the wake of a circular cylinder. These calculations quantify the nonlinear response of the system to three-dimensional perturbations near threshold for the two separate linear instabilities of the wake: mode A and mode B. The objectives are to classify the nonlinear form of the bifurcation to mode A and mode B and to identify the conditions under which the wake evolves to periodic, quasi-periodic, or chaotic states with respect to changes in spanwise dimension and Reynolds number. The onset of mode $\mathrm{A}$ is shown to occur through a subcritical bifurcation that causes a reduction in the primary oscillation frequency of the wake at saturation. In contrast, the onset of mode B occurs through a supercritical bifurcation with no frequency shift near threshold. Simulations of the three-dimensional wake for fixed Reynolds number and increasing spanwise dimension show that large systems evolve to a state of spatiotemporal chaos, and suggest that three-dimensionality in the wake leads to irregular states and fast transition to turbulence at Reynolds numbers just beyond the onset of the secondary instability. A key feature of these 'turbulent' states is the competition between self-excited, threedimensional instability modes (global modes) in the mode A wavenumber band. These instability modes produce irregular spatiotemporal patterns and large-scale 'spot-like' disturbances in the wake during the breakdown of the regular mode A pattern. Simulations at higher Reynolds number show that long-wavelength interactions modulate fluctuating forces and cause variations in phase along the span of the cylinder that reduce the fluctuating amplitude of lift and drag. Results of both two-dimensional and three-dimensional simulations are presented for a range of Reynolds number from about 10 up to 1000 .
\end{abstract}

\section{Introduction}

A fascinating feature of non-equilibrium fluid systems is the formation and destruction of spatial patterns. Pattern formation can be viewed as the signature of an underlying instability and often gives the initial clues to understanding the dynamics behind a complex system. Cross \& Hohenberg (1992) give a comprehensive review of pattern formation in hydrodynamics, nonlinear optics, chemical and biological systems. To a large degree the dynamics of these diverse physical systems can be described using similar concepts: linear instabilities, nonlinear saturation, mechanisms for pattern selection, and so forth. Elements of pattern formation are used in the present study as a framework for examining the sequence of global instabilities that develop in the wake of an infinitely long circular cylinder as it makes the transition 
from simple to chaotic dynamics with increasing Reynolds number. We can relate the linear instabilities of the ideal system to specific flow patterns observed in the wake and show that competition between these instability modes explains much of the complex behaviour observed in experiment. In many ways this scenario does not depend on details of the system geometry and should represent the development of complex dynamics in a number of similar free shear flows. Much of the focus in the present work will be on pattern destruction and the process that leads to irregular dynamics and 'turbulence' in the wake.

The two-dimensional vortex street in the wake of a circular cylinder is one of the most famous examples of pattern formation in fluids. It is known to result from a global Hopf bifurcation of the steady flow (Jackson 1987; Mathis, Provansal \& Boyer 1987; Zebib 1987). This bifurcation (the primary instability) occurs when the region of absolute instability in the near wake of the cylinder becomes sufficiently large. The basic pattern of two-dimensional vortex shedding dominates our conceptual view of the wake behind most bluff bodies. In the case of a circular cylinder, remnants of two-dimensional vortex shedding persist to extremely high Reynolds number and can still be observed when the wake is fully turbulent. Obviously the flow becomes more complex with increasing Reynolds number and the ideal two-dimensional vortex shedding pattern is disrupted by transition in the free shear layers in the near wake and eventually by turbulent transition in the boundary layer on the surface of the cylinder. However, the onset of 'turbulence' in the wake is an intrinsically threedimensional phenomenon that begins at a low Reynolds number with the absolute instability of the two-dimensional flow with respect to spanwise perturbations (the secondary instability).

Recent experiments have revealed a rich variety of pattern formation associated with the secondary instability of the Kármán vortex street and subsequent transition to turbulence. Roshko (1954) first identified the transition range for flow past a circular cylinder as the range of Reynolds number where velocity fluctuations become irregular. Early flow visualization studies by Hama (1957) and Gerrard (1978) linked those fluctuations with the development of 'waviness' in the spanwise vortices and Gerrard's ubiquitous 'fingers of dye.' However, it was Williamson (1988) who showed with great clarity the intricate structure of the three-dimensional cylinder wake in the transition range. The basic patterns consist of two types of three-dimensional vortex shedding that occur in a particular sequence as the Reynolds number is increased. Following the nomenclature introduced by Williamson (1988), we shall refer to these instabilities as mode A and mode B vortex shedding. Each flow pattern is centred around a different spanwise wavelength and is observed with different degrees of regularity. Quantitative visualization studies of the near wake by Mansy et al. (1994), Wu et al. (1996) and Brede, Eckelmann \& Rockwell (1996) established the variation of wavelength with Reynolds number and even provided some direct experimental measurements of the three-dimensional vorticity field. At the same time, Meiberg \& Lasheras (1988) showed that similar three-dimensional shedding modes develop naturally from perturbations in the plane wake behind a splitter plate, so there is good evidence that these phenomena represent instabilities in a broad family of free shear flows.

The origin of these patterns in the wake of an infinitely long cylinder may be understood by examining the linear instabilities of the ideal two-dimensional flow (Noack \& Eckelmann 1994; Barkley \& Henderson 1996). Like the onset of vortex shedding, the relevant instabilities are global and absolute, and tied to the instability of three-dimensional global modes in the wake. Huerre \& Monkewitz (1990) discuss 


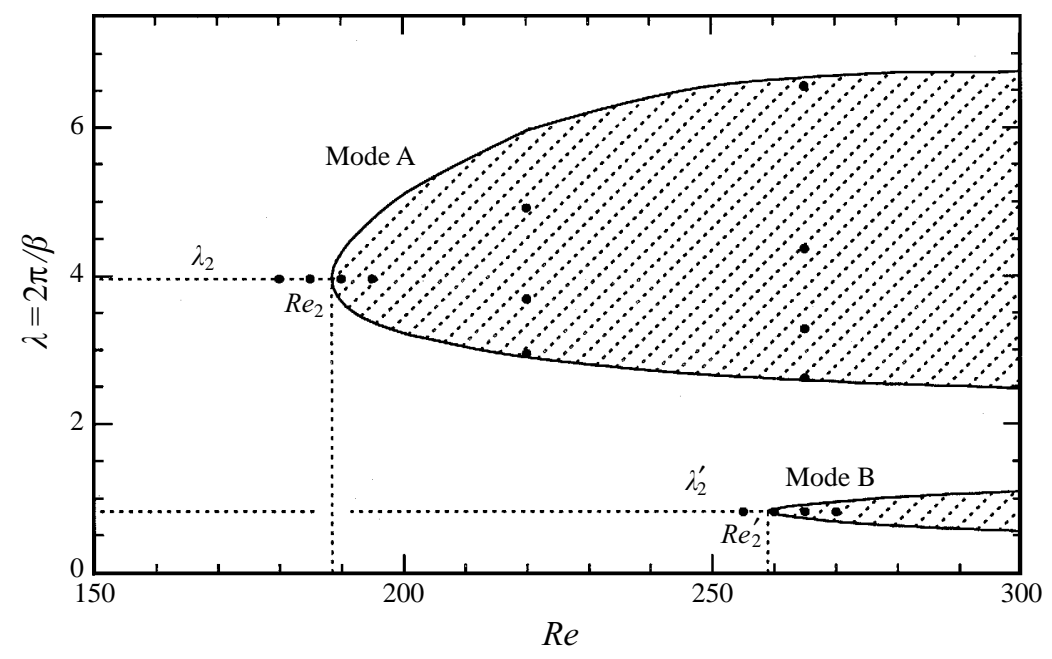

Figure 1. Curves of neutral stability for the two-dimensional Kármán vortex street with respect to spanwise perturbations. Linearly unstable modes exist everywhere inside the shaded regions. The upper region corresponds to a family of long-wavelength instabilities with critical values $R e_{2} \simeq 190$, $\lambda_{2} \simeq 3.96 d$; the lower region corresponds to a family of short-wavelength instabilities with critical values $R e_{2}^{\prime} \simeq 260, \lambda_{2}^{\prime} \simeq 0.822 d$. Each point $(\bullet)$ on this plot represents a three-dimensional mode considered in $\$ 3$.

these relatively new and somewhat controversial stability concepts in the context of spatially developing flows. The linear stability problem determines the structure and spatiotemporal symmetry of the global modes and the critical Reynolds number where they first become unstable to small perturbations. Once perturbed these modes are self-excited and cause transition to a three-dimensional state. The symmetry of the three-dimensional wake after transition is determined by the spatiotemporal symmetry of the destabilizing global mode. Barkley \& Henderson (1996) showed that the three-dimensional flow patterns observed in experiment are due to two separate linear instabilities, and that each instability leads to a distinct symmetry-breaking bifurcation from the ideal two-dimensional flow.

Figure 1 shows the neutral-stability curves for the wake, identifying the regions of instability that produce modes A and B. Parameters characterizing the wake instability are the Reynolds number $R e$ and the spanwise perturbation wavelength $\lambda$ (in units of cylinder diameter $d$ ) or wavenumber $\beta=2 \pi / \lambda$. For mode $\mathrm{A}$ the critical values are $R e_{2} \simeq 190$ and $\lambda_{2} \simeq 3.96 d$, while for mode B they are $R e_{2}^{\prime} \simeq 260$ and $\lambda_{2}^{\prime} \simeq 0.822 d$. There is now excellent agreement between computations based on the the linear theory and experimental measurements of the critical wavelength and Reynolds number (Barkley \& Henderson 1996; Williamson 1996a-c). There is also good experimental validation of the symmetry of the destabilizing modes predicted by the linear theory (Barkley \& Henderson 1996; Brede et al. 1996; Williamson 1996b). Simulations of the three-dimensional flow by Zhang et al. (1995), Thompson, Hourigan \& Sheridan (1996), and Henderson \& Barkley (1996) have reproduced the essential features observed in experiment and there is little doubt regarding the qualitative structure of the three-dimensional wake in the transition range.

What is not well understood is the scenario by which the wake develops irregular dynamics when the Reynolds number is increased beyond the secondary instability threshold. In order to look at possible scenarios leading to 'turbulence' in the cylinder 
wake, we will put aside for the moment the ideal problem of flow past an infinitely long cylinder in an unbounded domain and consider only systems with a finite spanwise dimension $L$. In computation $L$ represents the distance over which the velocity field is perfectly periodic (the size of the largest disturbances in the infinite system). In experiment $L$ may be thought of as roughly analogous to the aspect ratio of the system, but end effects introduce a fundamental and important difference. We also make an important conceptual assumption: that the dynamics may be described purely in terms of the global modes of the wake. This reduces the complexity of the three-dimensional wake to a one-dimensional system, the only important dimension represented by the character of the flow in the spanwise direction. Changes in the system dynamics can be characterized with respect to two numbers: the control parameter $R e$ (the Reynolds number), and the system size $L$ (the spanwise dimension). Experiments and computations show that for certain values of these parameters the flow has chaotic states, that is irregular behaviour that persists to long times even under constant external conditions. This is a manifestation of instability in a deterministic system and not of external noise. Chaotic behaviour is associated with systems that possess a large number of degrees of freedom which are excited as we go to the large-system limit $R e \rightarrow \infty, L \rightarrow \infty$. There are different possibilities depending on how we take these limits.

In the idealized problem one should study the transition from regular to chaotic dynamics by taking $L=\infty$ and looking at the sequence of bifurcations that occur with increasing control parameter $R e$. This is effectively impossible for computation unless we linearize about certain intermediate states. It is impossible in experiment although $L$ can in principle be taken sufficiently large that finite-size and end effects are small. $\dagger$ In a more realistic scenario we can reach chaotic states in two ways, starting from a system with a sufficiently complicated set of linear instabilities. The simplest is to fix the control parameter $R e$ and let $L \rightarrow \infty$. In many situations this leads to spatiotemporal chaos characterized by the interaction of a moderate number of modes in a dissipative system. Alternatively, we can fix the system size $L$ and let $R e \rightarrow \infty$. This is the regime of strong turbulence achieved by removing all dissipation from the system. To talk about the 'route to turbulence' in the wake we must distinguish between these two limits.

The present study focuses not so much on the limiting values of these parameters as on how they affect the transition to irregular states observed in experiment. Direct numerical simulation (DNS) is used to study three-dimensional flows that arise from perturbations to the two-dimensional wake in two ways: either for fixed $L$ and small variations in control parameter $R e$, or for fixed control parameter $R e$ and increasing system size $L$. In the latter case we follow the rationale given in $\$ 2.2$, increasing the original system size in powers of 2 in order to demonstrate changes in the dynamics due to the presence of more global modes, i.e. as we approach the continuous spectrum of the infinite problem. This particular sequence guarantees nested solution spaces where the dynamics of smaller systems are embedded within those of larger ones. Resolution is increased in proportion to $L$ to ensure that the largest discrete wavenumbers always lie in the dissipative part of the spectrum.

The neutral-stability diagram shown in figure 1 provides the necessary framework

$\dagger$ Finite-size effects are due to discretization of the continuous spectrum because of finite $L$, whereas end effects are due to the constant forcing of low-wavenumber modes by fluctuations at the ends of a finite-span cylinder. Generally speaking, computations are restricted by finite-size effects and experiments are polluted by end effects. 
for reducing a study of the entire $(R e, L)$-parameter space to a small number of focused questions. There are three important cases to understand in relation to transition. First is the nonlinear response of the system to perturbations near the critical point for mode $\mathrm{A}$ at $R e_{2}$ (\$3.3). In addition to providing the basic nonlinear form of the bifurcation, this also provides some qualitative information about all modes in the A-band. Likewise, the second important case is the nonlinear response of the system to perturbations near the critical point for mode B at $R e_{2}^{\prime}(\S 3.4)$. The dynamics are special near these critical points since nonlinearity locks the flow into a single symmetry-related mode. Finally, we look at how the wake responds away from these critical points when a variety of three-dimensional modes are excited (\$§3.5 and 3.6). In spite of the seeming complexity of the three-dimensional flow during transition, these calculations show that the dynamics are controlled by a small number of self-excited global modes in the wake. In each case the growth of these modes can be related to the formation of specific flow patterns during transition. We shall also see that nonlinearity leads directly to irregular states throughout the transition range, rather than through a sequence of further bifurcations. In a more qualitative sense, the simulations show the effect of large-scale structure in the wake on fluctuating forces and flow patterns at higher Reynolds number.

\section{Computational methods}

\subsection{Formulation}

We consider the motion of a viscous fluid past an infinitely long circular cylinder placed perpendicular to a uniform free stream. The fluid is assumed to have constant density $\rho$ and constant dynamic viscosity $\mu$. The idealized incompressible flow depends on three dimensional parameters: the cylinder diameter $d$, the free-stream speed $u_{\infty}$, and the kinematic viscosity of the fluid $v=\mu / \rho$. The only non-dimensional combination of these parameters is defined as the Reynolds number, $R e \equiv u_{\infty} d / v$, and this serves as the control parameter for the system. The problem may be described in dimensionless variables with $u_{\infty}$ and $d$ serving as the reference scales for velocity and distance. The state of the fluid at any time $t$ as it moves past the cylinder is determined by the velocity field $\boldsymbol{u}(x, y, z, t)$ and the pressure field $p(x, y, z, t)$. These fields are described in a coordinate system where $x$ is aligned with the freestream direction, $y$ is normal to the free stream, and $z$ is along the span of the cylinder.

Our main objective is to determine the nature of the flow dynamics as a function of $R e$. The evolution of the flow is described by the incompressible Navier-Stokes equations, written in non-dimensional form as

$$
\begin{gathered}
\frac{\partial \boldsymbol{u}}{\partial t}=-\boldsymbol{N}(\boldsymbol{u})-\frac{1}{\rho} \nabla p+\frac{1}{R e} \nabla^{2} \boldsymbol{u} \text { in } \Omega, \\
\nabla \cdot \boldsymbol{u}=0 \quad \text { in } \Omega .
\end{gathered}
$$

$N(\boldsymbol{u})$ represents the nonlinear advection term:

$$
\boldsymbol{N}(\boldsymbol{u}) \equiv(\boldsymbol{u} \cdot \nabla) \boldsymbol{u} .
$$

The computational domain $\Omega$ represents a region of three-dimensional space surrounding the cylinder that contains what is deemed to be the 'important' part of the flow.

The first step of the discretization is to reduce the problem on infinity to a problem on a domain of finite spanwise dimension $L$. In other words, we consider only those 
flows $\boldsymbol{u}(\boldsymbol{x}, t)$ that satisfy the periodicity requirement

$$
\boldsymbol{u}(x, y, z, t)=\boldsymbol{u}(x, y, z+L, t) .
$$

This is an important restriction on the solution space for moderate $L$ and some implications are discussed below. The three-dimensional spatially periodic field $\boldsymbol{u}$ can be projected exactly onto a set of two-dimensional Fourier modes $\hat{\boldsymbol{u}}_{q}$ as

$$
\hat{\boldsymbol{u}}_{q}(x, y, t)=L^{-1} \int_{0}^{L} \boldsymbol{u}(x, y, z, t) \mathrm{e}^{-\mathrm{i}(2 \pi / L) q z} \mathrm{~d} z .
$$

Likewise, the spanwise modes $\hat{\boldsymbol{u}}_{q}$ give the expansion of the velocity field in a Fourier series:

$$
\boldsymbol{u}(x, y, z, t)=\sum_{q=-\infty}^{\infty} \hat{\boldsymbol{u}}_{q}(x, y, t) \mathrm{e}^{\mathrm{i}(2 \pi / L) q z} .
$$

Substituting the Fourier expansion of the velocity field into the Navier-Stokes equations, we obtain a coupled set of equations for the Fourier modes. To simplify the notation, we define the scaled wavenumber $\beta_{q} \equiv(2 \pi / L) q$ and the $q$-dependent operators

$$
\tilde{\nabla} \equiv\left(\partial_{x}, \partial_{y}, \mathrm{i} \beta_{q}\right), \quad \tilde{\nabla}^{2} \equiv\left(\partial_{x}^{2}, \partial_{y}^{2},-\beta_{q}^{2}\right) .
$$

The evolution equation for the Fourier modes can then be written as

$$
\begin{gathered}
\frac{\partial \hat{\boldsymbol{u}}_{q}}{\partial t}=-\boldsymbol{N}_{q}(\boldsymbol{u})-\frac{1}{\rho} \tilde{\nabla} \hat{p}_{q}+\frac{1}{R e} \tilde{\nabla}^{2} \hat{\boldsymbol{u}}_{q} \quad \text { in } \Omega, \\
\tilde{\nabla} \cdot \hat{\boldsymbol{u}}_{q}=0 \quad \text { in } \Omega .
\end{gathered}
$$

The nonlinear advection term provides the coupling between all modes. We can denote this term by

$$
\boldsymbol{N}_{q}(\boldsymbol{u})=L^{-1} \int_{0}^{L} \boldsymbol{N}(\boldsymbol{u}) \mathrm{e}^{-\mathrm{i}(2 \pi / L) q z} \mathrm{~d} z .
$$

Dissipation becomes important at wavenumbers $\beta_{D} \sim R e^{1 / 2}$; at wavenumbers $\beta>\beta_{D}$ the equations are dominated by viscosity. These high-wavenumber modes contribute little to the dynamics of the flow at large scales because their energy is rapidly dissipated by viscosity. For an adequate description of the dynamics in a system with a given spanwise dimension $L$ we only need a finite set of $M$ Fourier modes to cover the range of scales from $\beta=0$ (the mean flow) to $\beta_{D}=(2 \pi / L) M \sim R e^{1 / 2}$, or $M=O\left(L R e^{1 / 2}\right)$. We take as our final representation of the velocity field the truncated expansion

$$
\boldsymbol{u}(x, y, z, t)=\sum_{q=-M}^{M} \hat{\boldsymbol{u}}_{q}(x, y, t) \mathrm{e}^{\mathrm{i}(2 \pi / L) q z} .
$$

Equations (2.1) and (2.2) are simply alternative ways to describe the flow. Computationally it is more convenient to follow the evolution of the two-dimensional Fourier modes $\hat{\boldsymbol{u}}_{q}(x, y, t)$ than the full three-dimensional field $\boldsymbol{u}(\boldsymbol{x}, t)$. Because $\boldsymbol{u}$ is real, the Fourier modes satisfy the symmetry $\hat{\boldsymbol{u}}_{-q}=-\hat{\boldsymbol{u}}_{q}^{*}$. Therefore, only half of the spectrum $(q \geqslant 0)$ is needed. In addition to convenience, the Fourier representation of the velocity field has other intrinsic advantages. It provides a direct way of linking particular modes of the system with specific three-dimensional spatial patterns. Linear stability theory can predict which modes will have the strongest interaction with the 
two-dimensional flow to produce these patterns. The time-averaged amplitude of the Fourier modes gives a direct indication of how well-resolved the calculations are. And finally, the time-dependent amplitude of the Fourier modes provides a convenient way of explaining the transfer of energy to different scales in the three-dimensional wake.

\subsection{Subspaces and the approach to infinity}

Although our goal is to study the flow past an infinitely long cylinder, this is clearly not possible in a simulation of the full Navier-Stokes equations. Periodic boundary conditions are often used in computational fluid dynamics to approximate the flow on an infinite domain, but this is a false assumption. Periodic boundary conditions do not reproduce the same dynamics unless the dimension of the system in the periodic direction is large enough to provide a good representation of the continuous spectrum of the infinite problem. Keep in mind that the only admissible wavelengths are those which are consistent with the boundary conditions: $\lambda_{q}=L / q$. Even 'random noise' introduced in a computation with periodic boundary conditions can only excite a discrete set of modes with these wavelengths. Choosing $L$ too small can exclude important instability modes altogether or it can simply exclude the modal interaction that leads to complex behaviour in large systems. Here we suggest a rationale for exploring the dynamics of the infinite problem by examining the dynamics in a particular sequence of successively larger systems with carefully chosen initial conditions.

We begin by considering the effect of periodic boundary conditions on the space of possible solutions. For a given periodic length $L$, the velocity field $\boldsymbol{u}$ lies within a subspace spanned by the Fourier modes $\hat{\boldsymbol{u}}_{q}$. We can write this as follows:

$$
\boldsymbol{u}(x, y, z, t) \in \mathscr{S}^{L}=\operatorname{span}\left\{\hat{\boldsymbol{u}}_{q}(x, y, t) \mathrm{e}^{\mathrm{i}(2 \pi / L) q z}, \quad q=0, \pm 1, \ldots\right\} .
$$

The Navier-Stokes equations preserve this subspace, meaning that the state which evolves from an initial condition in $\mathscr{S}^{L}$ will always remain there - the flow itself cannot generate larger scales. Because of the Fourier expansion of the velocity field, a sequence of larger subspaces can be nested in the sense that

$$
\mathscr{S}^{L} \subset \mathscr{S}^{2^{n} L} \subset \mathscr{S}^{\infty} .
$$

This means that every mode $\hat{\boldsymbol{u}}_{q}$ in $\mathscr{S}^{L}$ is also a mode of $\mathscr{S}^{2^{n} L}$, so that as we increase $L$ in powers of 2 we are adding new degrees of freedom while retaining all degrees of freedom of the smaller systems. As long as we follow this sequence, the final state in each small system lies within an exact (stable or unstable) subspace of all larger systems. This is the most precise way to study the approach to the dynamics of the infinite problem.

Fluctuations at the ends of a finite-span cylinder in experiments set the scale of the largest disturbances and provide a sustained excitation at low wavenumbers. Nonlinearity guarantees that all modes will be excited to some degree. The situation is quite different in computation. In the light of (2.3) we may adopt the computational point of view that a spanwise-periodic perturbation at wavelength $\lambda$ defines the effective spanwise dimension $(L \equiv \lambda)$ because there is no mechanism within the Navier-Stokes equations to generate larger scales. The initial conditions are the critical factor rather than the imposed periodic boundary conditions. From (2.4) we can see that the same initial condition will evolve to the same final state in the infinite system because the flow $\boldsymbol{u}(\boldsymbol{x}, t)$ always remains within the finite-dimensional subspace $\mathscr{S}^{L}$. The resulting spatially periodic flow may be a valid solution to the NavierStokes equations in an 'infinite' domain, but it is physically irrelevant unless we can 


$\begin{array}{ccrrl}\text { mesh } & K & L_{i} & L_{o} & d / 2 L_{i} \\ M_{1} & 148 \text { to } 170 & 8 & 24 & 0.0625 \\ M_{2} & 166 \text { to } 175 & 16 & 32 & 0.03125 \\ M_{3} & 182 \text { to } 201 & 32 & 48 & 0.015625\end{array}$

TABLE 1. Domain size parameters for the small, medium, and large computational domains shown in figure 2. The factor $d / 2 L_{i}$ is the blockage (fraction of the cross-sectional area $2 L_{i} L$ obstructed by the cylinder area $d L$ ).

also show it is stable in the presence of longer wavelength disturbances, i.e. in each larger subspace. The present study will show that, for fixed Reynolds number, 'small' systems $(L \approx 1 d)$ give rise to time-periodic behaviour while 'large' systems $(L \approx 10 d)$ evolve to a state of spatiotemporal chaos. Because the dynamics are controlled by the interaction of a small number of instability modes, computations with moderate $L$ are sufficient for deducing what happens in the limit $L \rightarrow \infty$.

\subsection{Implementation}

The fully discrete solution relies on the representation of the Fourier modes, $\hat{\boldsymbol{u}}_{q}(x, y, t)$ and $\hat{p}_{q}(x, y, t)$, by a non-conforming spectral element method. The formulation for non-conforming elements was introduced by Bernardi et al. (1992) as an extension to the original spectral element method developed by Patera (1984). In the spectral element method the computational domain is partitioned into $K$ non-overlapping elements, and within each element both the solution and geometry are represented by a high-order polynomial basis. In two or more dimensions this basis can be constructed as a product of one-dimensional polynomials. The current implementation uses curvilinear quadrilateral elements and relies exclusively on Gauss-Lobatto Legendre polynomials to represent the discrete solution. Any two-dimensional field is described by $K N^{2}$ numbers, where $N$ is the number of basis coefficients in each direction. Since the complex vector field $\hat{\boldsymbol{u}}_{q}(x, y, t)$ has six scalar components (three real and three imaginary), it requires $6 \times K N^{2}$ numbers. The full three-dimensional vector field $\mathbf{u}(\boldsymbol{x}, t)$ is expanded over $2 M+1$ such modes, but because of the symmetry of these modes the discrete representation only requires a total of $6 \times M K N^{2}$ coefficients. Likewise, the pressure field $p(x, t)$ is described by $2 \times M K N^{2}$ coefficients. The number $n_{\mathrm{dof}} \simeq 2 \times M K N^{2}$ is equivalent to the number of grid points in physical space needed to represent all degrees of freedom in a three-dimensional scalar field. In the present set of calculations, $n_{\mathrm{dof}} \approx 10^{6}$ grid points.

Figure 2 shows the basic computational domains used for simulations of the wake over the entire range of Reynolds number and table 1 shows the domain size parameters. The formulation described above can be used for two-dimensional simulations by solving only for the zeroth Fourier mode. Simulations of the threedimensional flow use the same computational domain for all Fourier modes. Although the Fourier modes could alternatively be discretized by introducing a finite difference grid or a collection of finite elements, the non-conforming method used here allows for local mesh refinement while preserving the block structure of the calculations. The computational domains shown in figure 2 represent only the base mesh and calculations at various Reynolds numbers incorporate some refinement of the near wake. The use of a non-conforming mesh to simulate the flow at lower Reynolds number is helpful but not critical. Beyond the details of the polynomial basis and the treatment of non-conforming elements in the mesh, the method follows a standard 

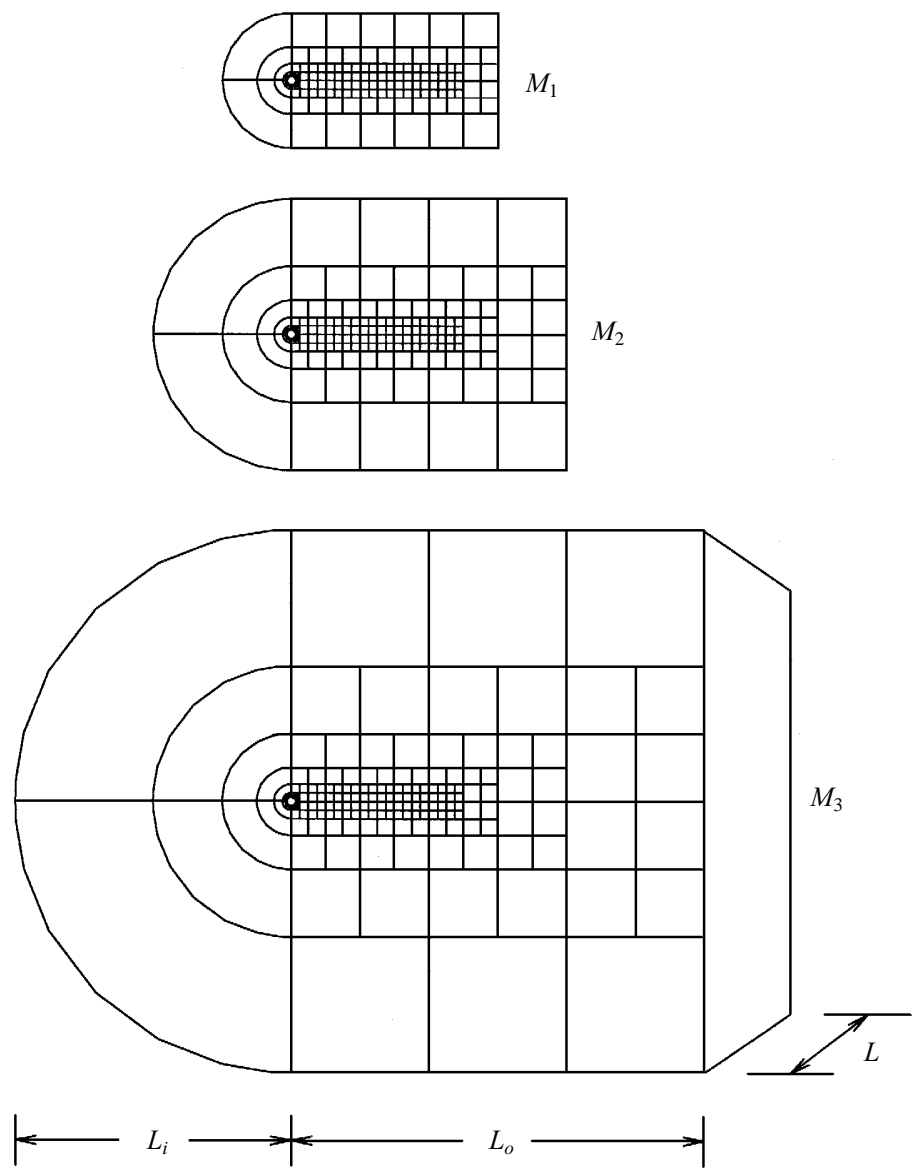

Figure 2. Computational domains used in the present study. Each domain is a subset of the largest. The parameters $L_{o}$ and $L_{i}$ determine the cross-sectional size, and $L$ determines the spanwise dimension. Each computational domain is divided into $K$ elements, and within each element the solution and geometry are represented by $N^{2}$ polynomial coefficients. The three-dimensional flow is decomposed into $M$ Fourier modes in the periodic spanwise direction.

Galerkin finite element procedure to discretize equation (2.2). Further details of this particular approach for non-conforming elements are described by Henderson \& Karniadakis (1995).

Boundary conditions are required for the velocity and pressure fields on all exterior boundaries of $\Omega$, including the surface of the cylinder. For convenience these are specified in physical rather than Fourier space. At the surface of the cylinder the fluid velocity satisfies the no-slip condition: $\boldsymbol{u}(\boldsymbol{x}, t)=(0,0,0)$. Far away from the cylinder and outside the wake, it matches the free-stream velocity: $\boldsymbol{u}(\boldsymbol{x}, t)=(1,0,0)$. Across the outflow plane downstream the approximate boundary condition for the velocity field is $\partial_{x} \boldsymbol{u}(\boldsymbol{x}, t)=(0,0,0)$. At this same location the pressure is set to a constant value of zero. Along all other boundaries the pressure satisfies the high-order Neumann boundary condition given by Karniadakis, Israeli \& Orszag (1991). This condition, which is derived by requiring the pressure field to satisfy the normal component of the momentum equation along the boundary, helps control divergence errors introduced by the splitting scheme described below.

The location of external boundaries affects quantities derived from the simulations 
such as shedding frequency and drag. The detailed convergence study presented by Barkley \& Henderson (1996) was used as the principal guide in selecting an appropriate size and resolution. The smallest domain, $M_{1}$, is somewhat larger than domains used in similar calculations of the three-dimensional flow over this range of Reynolds number (Karniadakis \& Triantafyllou 1992; Zhang et al. 1995; Thompson et al. 1996). Even this domain produces an acceptable quantitative simulation of the flow. In order to reduce the blockage effect on $M_{1}$ the constant-velocity boundary conditions were replaced with a condition of periodicity for both velocity and pressure along the upper and lower boundaries of the wake region, i.e. $\boldsymbol{u}\left(x, y-L_{i}, z, t\right)=$ $\boldsymbol{u}\left(x, y+L_{i}, z, t\right)$ for $x \geqslant 0$. Results for all three domains agree to better than $2 \%$. The effect of spanwise dimension $L$ is examined directly in $\S 3$.

The set of modal equations are integrated forward in time using the three-step splitting scheme described by Karniadakis et al. (1991). This time-stepping algorithm replaces equation (2.2) by a sequence of steps where the nonlinear terms are computed explicitly while the pressure and diffusion terms are treated implicitly. Each implicit step requires the solution of an elliptic boundary-value problem as described below. The algorithm is essentially a projection method with a consistent pressure boundary condition that yields (in practice) second-order time accuracy. The explicit treatment of the nonlinear terms dictates the maximum allowable time step through a CFL-type condition, $(\Delta t / \Delta x)|\boldsymbol{u}|_{\max } \leqslant$ const. $\approx 0.72$. In all of the calculations $|\boldsymbol{u}|_{\max }=O(1)$, the minimum grid size is $\Delta x \approx 0.007$, and the corresponding maximum time step is $\Delta t \approx 0.005$.

Discretizing the implicit part of the time integration produces a set of linear systems for the discrete pressure and velocity fields that must be solved at each time step. Since both the real and imaginary parts of the complex pressure field satisfy the same algebraic system of equations, there are only $M$ systems to form ( $2 M$ to solve). The three components of the velocity vector also satisfy the same system of equations, giving $M$ additional systems to form (6M to solve). Each system is described by a real-valued matrix that is symmetric and positive-definite with a rank of $O\left(K N^{2}\right)$. Each matrix is reduced by factoring into Schur-complement form to eliminate rows and columns associated with element interiors. This reduced system for the boundary points has a rank of $O(K N)$ and can be solved directly from the $L U$ factorization of the Schur-complement matrix. Once the boundary solution is known, the solution on the interior of each element can be updated by solving $K$ smaller systems of rank $O\left(N^{2}\right)$. The Schur-complement factorization is crucial for the direct solution of the large matrix systems associated with spectral element methods. Factorization is performed once at the beginning of the calculation, and time stepping only requires back-substitution using the stored matrices. The procedure is identical for each of the Fourier modes and equivalent to solving $2 M$ independent two-dimensional problems with real data.

The repetition of calculations for each of the Fourier modes suggests a natural strategy for distributing the computational work over a collection of parallel processors: data for each mode is assigned to a different computer. Time integration is carried out for each mode in parallel with an exchange of data at the beginning of the time step to evaluate the nonlinear term. This term is computed pseudo-spectrally on a grid of points in physical space through use of the fast Fourier transform (FFT). The nonlinear calculation was not dealiased and although there is some pollution of high-wavenumber modes this is not a problem at moderate Reynolds number. During the FFT data are exchanged (the Fourier components) among all processors in the algorithmic equivalent of a parallel matrix transpose. This communications kernel is 
common to most parallel implementations of spectral methods (see e.g. Karniadakis $\&$ Orszag 1993). Evaluating the nonlinear term accounts for roughly one-quarter of the computational work. The remaining work goes toward solving the linear systems in the pressure and diffusion steps. These calculations require no interaction between modes and they proceed in parallel with the work perfectly balanced across the collection of parallel processors.

The strategy outlined above was implemented on two dedicated parallel computers: the Intel Paragon and the Cray T3D. Both of these machines offer relatively fast compute nodes and a custom communications network with low latency and high bandwidth. The data exchange needed to evaluate the nonlinear term was performed via explicit message passing. The code communicates through a limited set of drivers so that porting to new message passing systems is relatively easy, i.e. only new communication drivers need to be written. For example, after developing the code on the Paragon, porting it to the T3D only took a few hours. The largest calculations performed as part of the current study involve $8 \times M K N^{2} \approx 6$ to 7 million unknowns per time step and 2 to 3 gigabytes worth of run-time data (field data and stored matrices). Distributed over 64 processors, these calculations take approximately $5 \mathrm{~s}$ per time step on either machine. Simulations at this resolution would have been impractical without access to large-scale parallel computing systems.

\section{Results}

\subsection{Overview}

The present study encompasses a wide range in Reynolds number from $R e \approx 10$ (steady two-dimensional flow) to $R e=1000$ (turbulent three-dimensional flow). We begin with an overview of changes in the flow dynamics with increasing Reynolds number, concentrating on the response of wake oscillation frequency (shedding frequency) and mean drag coefficient. Both are unique functions of Reynolds number along the two-dimensional branch of solutions. From an ensemble of experimental measurements, their values at low Reynolds number are currently known to within an accuracy of about $5 \%$, although the experimental shedding frequency curve in the two-dimensional range is now believed to be accurate to better than $1 \%$ (Williamson $1996 a$ ). Both shedding frequency and drag show distinct changes at the various bifurcation points of the wake and can be used as a guide to interpreting changes in the wake structure and dynamics as a function of Reynolds number.

In non-dimensional form the shedding frequency is referred to as the Strouhal number. It is defined as $S t \equiv f d / u_{\infty}$, where $f$ is the peak oscillation frequency of the wake. Shedding frequency measurements in the laboratory are typically made using hot-wire signals from the unsteady velocity field near the edge of the separating shear layer. However, because vortex shedding is associated with a global instability, any signal derived from measurements of the flow carries the signature of the vortex shedding frequency. Shedding frequencies presented here are generally determined from the zero-crossing points of the unsteady lift signal. Shedding frequency can be monitored as a direct indication that the unsteady flow is converging to a limit cycle.

The Strouhal-Reynolds number relationship is shown in figure 3. At low Reynolds number the flow is steady $(S t=0)$ and symmetric about the centreline of the wake. At $R e_{1} \simeq 47$ the steady flow becomes unstable and bifurcates to a two-dimensional, time-periodic flow. The shedding frequency of the two-dimensional flow increases smoothly with Reynolds number along the curve shown in figure 3. Note that each 


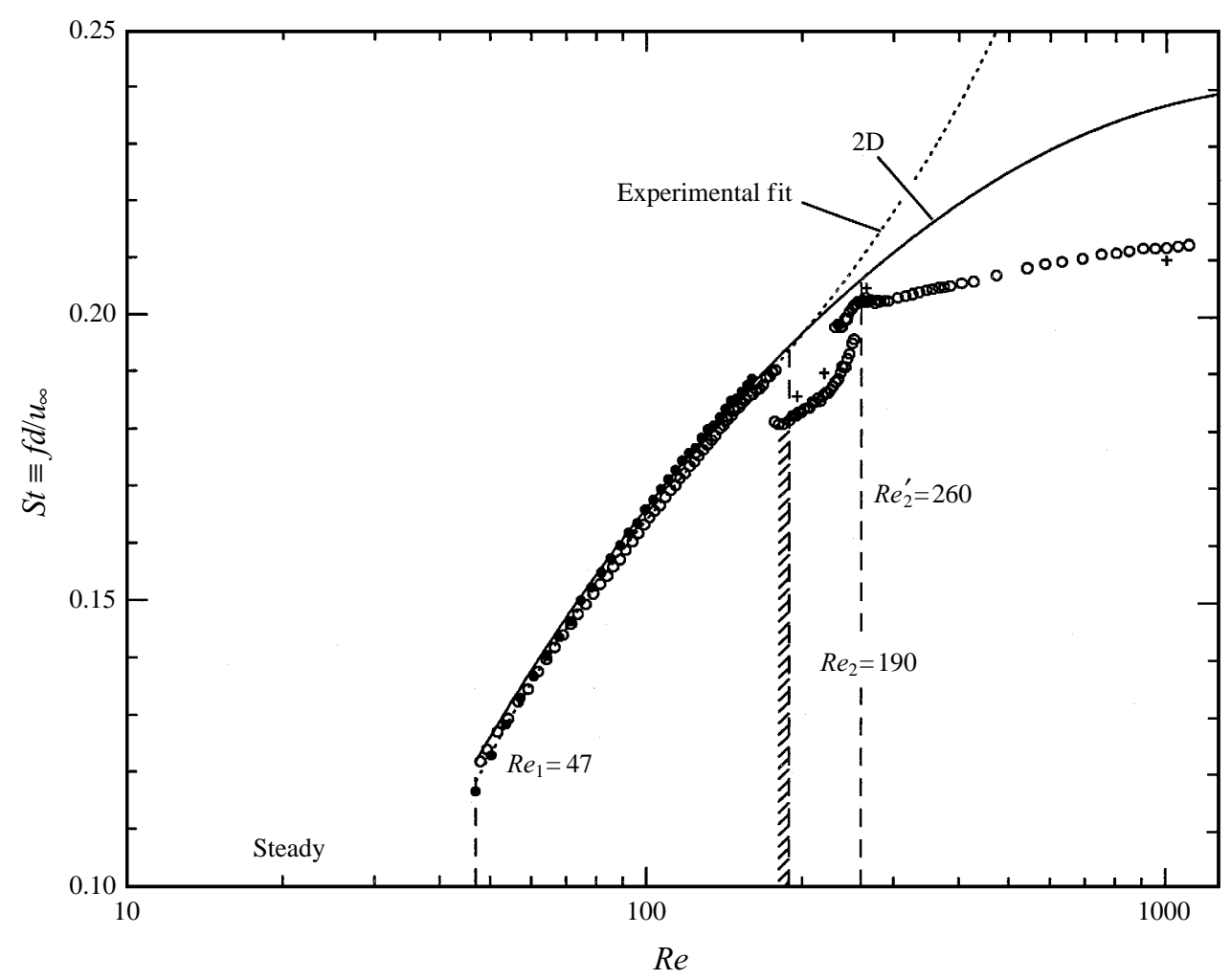

FigURE 3. Variation of wake oscillation frequency (Strouhal number) with Reynolds number for the flow past a circular cylinder, from experimental measurements and computer simulations: $\circ$, Williamson (1989); •, Hammache \& Gharib (1991); +, three-dimensional simulations from the present study; the solid line is a curve fit to two-dimensional simulation data for $R e$ up to 1000 Dashed lines mark the critical Reynolds number for various wake instabilities. The shaded area indicates a subcritical range described in $\$ 3.3$ where the wake is unstable to finite-amplitude perturbations.

point along the two-dimensional curve represents a perfectly time-periodic flow and there is no evidence of further two-dimensional instabilities for Reynolds numbers up to $R e \approx 1000$. At $R e_{2} \simeq 190$ the two-dimensional wake becomes absolutely unstable to long-wavelength spanwise perturbations and bifurcates to a three-dimensional flow (mode A). As the wake passes through the bifurcation point at $R e_{2}$, experiments indicate that there are two important changes in the shedding frequency: (i) there is a sharp drop in magnitude, and (ii) above $R e_{2}$ the flow is no longer time-periodic but oscillates within a broad band of frequencies. Experimental frequency measurements shown in figure 3 which are not along the two-dimensional branch represent the dominant peak in those broad-band spectra. There is a direct relationship between the drop in frequency and the onset of mode A; we return to this point in §3.3. The other feature of the frequency curve relevant to the present study is the change in slope at $R e_{2}^{\prime} \simeq 260$ which coincides with the linear instability of mode B. Although mode $\mathrm{B}$ is observed in experiments at Reynolds number as low as $R e \approx 200$, there is clearly a measurable change in shedding frequency at $R e_{2}^{\prime}$. Note that three-dimensional calculations follow the experimental trend for $R e>R e_{2}$.

Figure 4 shows the drag curve for flow past a circular cylinder for Reynolds numbers up to 1000 . In the computations the spanwise-averaged fluid force $\boldsymbol{F}(t)$ is 


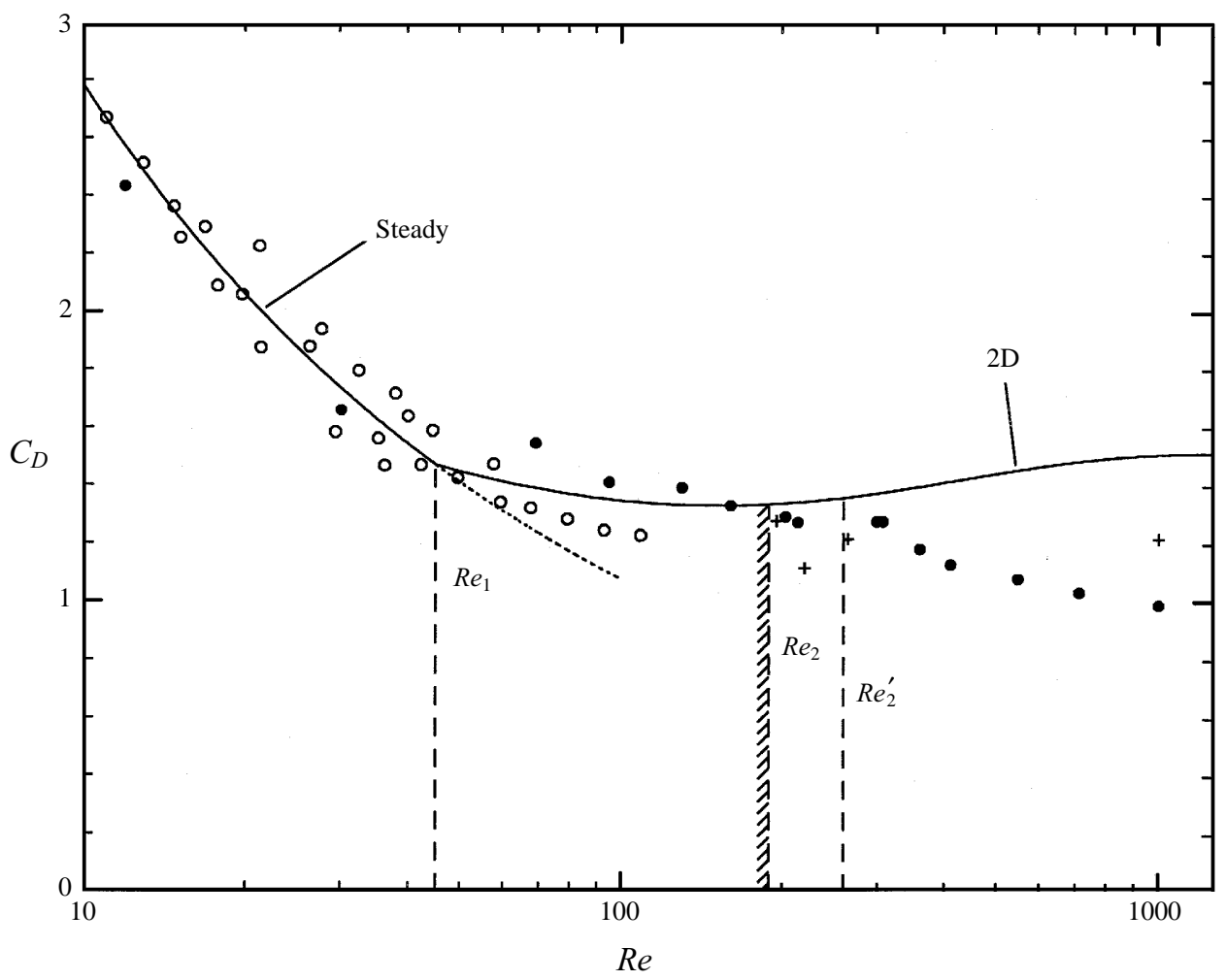

FiguRE 4. Drag coefficient as a function of Reynolds number for the flow past a circular cylinder, from experimental measurements and computer simulations: $(\circ, \bullet)$, Wieselsberger $(1921) ;+$, three-dimensional simulations from the present study; the solid line is a curve fit to two-dimensional simulation data for $R e$ up to 1000 .

computed by integrating the shear stress and pressure over the surface of the cylinder. The $x$-component of $\boldsymbol{F}$ is the drag, the $y$-component is the lift. The force is normalized by the free-stream dynamic pressure and the projected area of the body to produce force coefficients. The drag coefficient is defined as $C_{D}(t) \equiv F_{x}(t) / \frac{1}{2} \rho u_{\infty}^{2} d L$. The mean drag $C_{D}$ is simply the time-averaged value of $C_{D}(t)$. Because $C_{D}$ is determined from an average over the surface of the cylinder, it is much less sensitive to changes in the character of the wake at low Reynolds number than single-point measurements like the shedding frequency. The 'textbook' version of the drag curve is generally plotted on a log-log scale where the only discernible feature is the drag crisis at $R e=O\left(10^{5}\right)$. The flat response of $C_{D}$ to changes in Reynolds number is compounded by the fact that experimental drag measurements are extremely difficult to make at low Reynolds number, and subtle details of the drag curve are lost in the experimental scatter. The decrease in magnitude of $C_{D}$ in the steady regime can be fitted to a power-law curve and also makes a sharp but continuous transition at $R e_{1}$. Henderson (1995) gives the form and coefficients for the steady and unsteady drag curves. At the onset of vortex shedding about $1 / 3$ of the total drag force is due to skin friction from the boundary layer and $2 / 3$ is due to pressure drag. With increasing Reynolds number the skinfriction component continues to drop off while the pressure drag steadily increases. At higher Reynolds number the drag is due almost entirely to the variation in pressure around the surface of the cylinder. From $R e_{1}$ to $R e_{2}$ the drop in skin friction and 
increase in pressure drag almost cancel so that the $C_{D}-R e$ curve is relatively flat in this range. Experiments do not indicate a substantial change in the drag curve at $R e_{2}$ but there is a sharp drop away from the two-dimensional curve beginning at $R e_{2}^{\prime}$. Qualitatively, the drag and shedding frequency curves show similar behaviour but the changes in $C_{D}$ are more subtle. Three-dimensional calculations also show a decrease in drag for $R e>R e_{2}$, but there are not enough experimental measurements for a detailed comparison.

\subsection{Nonlinear dynamics and 'global modes' of the wake}

Everything that follows is based on the concept that spatially developing flows support self-excited global modes. Common examples of such flows include wakes, jets, and shear layers. In the case of flow past a circular cylinder we can represent the threedimensional flow near the secondary instability threshold as a combination of two global modes. These modes represent the saturated primary instability $\left(\phi_{0}\right)$ and a secondary instability mode $\left(\phi_{1}\right)$ :

$$
\boldsymbol{u}(\boldsymbol{x}, t)=U(t) \phi_{0}(\boldsymbol{x}, t)+A(t) \phi_{1}(\boldsymbol{x}, t) .
$$

$\phi_{0}$ and $\phi_{1}$ are assumed to be time-periodic functions with unit norm; $U(t)$ and $A(t)$ give their time-dependent amplitudes. In the linear approximation $\phi_{1}$ is simply the leading eigenfunction of a temporal (Floquet) stability problem with given real wavenumber $\beta$ and growth rate $\sigma(\beta) . \dagger$ The local modes of the system would be the eigenfunctions of the one-dimensional Floquet stability problem associated with the time-periodic base flow at a given streamwise location. All local modes with a given $\beta$ are contained in the global mode for that $\beta$.

Our first goal is to understand the effect of small perturbations to the twodimensional flow. By definition the primary instability $\boldsymbol{\phi}_{0}$ is at a finite-amplitude equilibrium state $U(t)=U_{0}$. For $|A| \ll\left|U_{0}\right|$ the amplitude of the secondary instability is given by $A(t) \propto \exp \sigma t$, and $\phi_{1}$ takes the form

$$
\boldsymbol{\phi}_{1}(\boldsymbol{x}, t)=\hat{\boldsymbol{\phi}}_{1}(x, y, t) \mathrm{e}^{\mathrm{i} \beta z}+\hat{\boldsymbol{\phi}}_{1}^{*}(x, y, t) \mathrm{e}^{-\mathrm{i} \beta z} .
$$

Now assume that the system responds such that $\sigma(\beta)<0$ for $R e<R e_{c}$ and $\sigma(\beta)=0$ for $R e=R e_{c}, \beta=\beta_{c}$. We define the reduced control parameter $\epsilon \equiv\left(R e-R e_{c}\right) / R e_{c}$ to characterize small variations from the critical value $R e_{c}$. For parameter values $\epsilon<0$ the flow is linearly stable, whereas for $\epsilon=0$ there is a pattern-forming instability that sets in at finite wavenumber $\beta=\beta_{c}$. For $\epsilon>0$ the infinite system has a continuous band of wavenumbers $\beta_{-}<\beta_{c}<\beta_{+}$for which the flow is unstable. Small perturbations near the critical point $\left(R e_{c}, \beta_{c}\right)$ will result in a three-dimensional flow pattern that grows everywhere in space with a structure determined by $\phi_{1}$. Although we cannot write down an explicit form for $\phi_{1}$, we can identify the patterns formed due to its presence in the flow. Linear theory is enough to predict the critical Reynolds number $\left(R e_{c}\right)$ and pattern wavelength $\left(\lambda_{c}=2 \pi / \beta_{c}\right)$, but nonlinear effects must be included if we want to understand the dynamics of the flow beyond the mere onset of the linear instability.

A low-dimensional dynamical systems approach is a natural way to analyse globally unstable flows. Close to a critical point we can reduce the dynamics of the threedimensional wake to those of a discrete-time dynamical system. We take an approach

$\dagger$ In general $\sigma$ will be complex, but Barkley \& Henderson (1996) show that the most unstable modes of the wake all have real $\sigma$. Here we interpret the complex frequency $\sigma$ as a real (temporal) growth rate. 
similar to the one above, fixing the perturbation wavelength $\lambda=\lambda_{c}$ and writing the growth rate as $\sigma=\sigma(\epsilon)$. Next we discretize time by only examining the state of the system at discrete times $t_{n}$ representing one pass through the shedding cycle. The global modes are time-periodic, $\boldsymbol{\phi}_{i}\left(\boldsymbol{x}, t_{n+1}\right)=\boldsymbol{\phi}_{i}\left(\boldsymbol{x}, t_{n}\right)$, so the dynamics are described largely by the discrete-time evolution of their amplitudes, $U_{n} \equiv U\left(t_{n}\right)$ and $A_{n} \equiv A\left(t_{n}\right)$. Our goal is to model the Navier-Stokes equations with a simple nonlinear equation for these amplitudes.

As the instability grows the linear superposition (3.1) no longer holds. For $|\epsilon| \ll$ 1 and $\left|A_{n}\right| \ll\left|U_{0}\right|$ the flow can be represented as an expansion about the twodimensional state in powers of the amplitude $A_{n}$. The form of that expansion may be deduced directly from the nonlinear form of the Navier-Stokes equations. Changes in the amplitude of $\phi_{0}$ as it transfers energy to $\phi_{1}$ are given by

$$
U_{n}=U_{0}-\sum_{j=1}^{\infty} \alpha_{0 j} A_{n}^{2 j} .
$$

Near the linear instability the evolution of $A_{n}$ will be given by $A_{n+1}=\mu_{1} A_{n}$, where $\mu_{1}=\exp \sigma T_{n}$ is the discrete-time linear growth rate and $T_{n}$ is the length of period number $n$. Nonlinearity eventually arrests the exponential growth and the long-time evolution of $A_{n}$ is given by

$$
A_{n+1}=\left(\mu_{1}-\sum_{j=1}^{\infty} \alpha_{1 j} A_{n}^{2 j}\right) A_{n} .
$$

Note that each of the constants $\mu_{1}$ and $\alpha_{i j}$ is some function of $\epsilon$. Near the critical point the variation of the linear growth rate is approximately $\mu_{1}=1+\mu_{1}^{\prime} \epsilon$, where $\mu_{1}^{\prime} \equiv \mathrm{d} \mu_{1} / \mathrm{d} \epsilon$. In this same regime the $\alpha_{i j}$ will be assumed constant since their variations are $O\left(\epsilon^{2}\right)$ or smaller. For a given initial state $\left(U_{0}, A_{0}\right)$, the amplitude equations describe the behaviour of transients $\left(U_{n}, A_{n}\right)$ and identify the finite-amplitude states $\left(U_{\infty}, A_{\infty}\right)$ which the flow evolves to at long times. Experiments or simulations of the full Navier-Stokes equations are needed to determine the values of the nonlinear coefficients.

Nonlinear classification of the bifurcation $\left(U_{0}, 0\right) \rightarrow\left(U_{\infty}, A_{\infty}\right)$ depends on the sign of $\alpha_{11}$, also called the Landau constant. Positive $\alpha_{11}$ corresponds to a supercritical or soft bifurcation. In this case the transition is continuous and the flow is stable below the critical point $(\epsilon<0)$. Negative $\alpha_{11}$ signifies a subcritical or hard bifurcation. In this case the transition is discontinuous and hysteretic because the flow is unstable to finite-amplitude perturbations below the critical point $(\epsilon \leqslant 0)$. To a large degree the distinction depends on the value of the critical wavenumber $\beta_{c}$ relative to the dissipation range $\beta_{D}$ for the system. This classification can be made precisely by studying the evolution of small perturbations with careful experiments or highresolution computer simulations of the full nonlinear system.

In each of the calculations presented here we take initial conditions of the form (3.1). The mode $\boldsymbol{\phi}_{0}$ is computed by integrating the two-dimensional Navier-Stokes equations in a given domain until the flow converges to a limit cycle with amplitude $U_{0}$. For a given wavelength $\lambda$, the mode $\phi_{1}$ is set to the leading eigenmode of the temporal stability problem. The initial amplitude of $\phi_{1}$ is chosen so that $\left|A_{0}\right| \approx 0.005\left|U_{0}\right|$, i.e. less than a $1 \%$ perturbation to the base flow. The evolution of the resulting (unstable) flow is then computed by integrating the full three-dimensional Navier-Stokes equations 
in a periodic domain capable of representing the initial perturbation and $M$ of its higher harmonics. The wavelength of the initial perturbation determines the spanwise dimension of the system, $L=\lambda$.

In the discrete nonlinear system the three-dimensional structure of the global modes is represented by a Fourier series expansion. Since $\phi_{0} \propto \hat{\boldsymbol{u}}_{0}$ and $\phi_{1} \propto \hat{\boldsymbol{u}}_{1}$, the amplitude of a global mode at later times can be evaluated directly from the amplitude of its fundamental Fourier mode:

$$
\begin{aligned}
& \left|U_{n}\right|^{2}=\frac{4}{\pi d^{2} u_{\infty}^{2}} \int_{\Omega}\left|\hat{\boldsymbol{u}}_{0}\left(x, y, t_{n}\right)\right|^{2} \mathrm{~d} \Omega, \\
& \left|A_{n}\right|^{2}=\frac{4}{\pi d^{2} u_{\infty}^{2}} \int_{\Omega}\left|\hat{\boldsymbol{u}}_{1}\left(x, y, t_{n}\right)\right|^{2} \mathrm{~d} \Omega .
\end{aligned}
$$

Although the full representation of $\phi_{1}(\boldsymbol{x}, t)$ involves higher harmonics for $t>t_{0}$, nonlinearity locks these modes to the fundamental $\hat{\boldsymbol{u}}_{1}$. Such modes are said to be passive or slaved. In a pure bifurcation the fundamental mode carries the largest component of the instability and is sufficient for tracking the amplitude. Note that the definition of amplitude in (3.4) depends on the computational domain $\Omega$, and therefore the nonlinear coefficients $\alpha_{i j}$ also depend on $\Omega$. However, our primary concern is with the sign (positive or negative) of $\alpha_{11}$, and this is independent of how the norm is defined.

\subsection{Bifurcation to mode $A$ : $R e \simeq 190, L=3.96 d$}

Nonlinear calculations for the precise onset of the secondary instability were first reported by Henderson \& Barkley (1996). Some of those results are included here for completeness. Figure 5 shows the growth and nonlinear saturation of a small perturbation to the wake at $R e=195, \lambda=\lambda_{2}=3.96 d$. The evolution of the threedimensional flow was computed on the small domain $M_{1}$ using $M=16$ modes. After $O(100)$ shedding periods the instability saturates. Near the point of saturation the instability grows faster than the exponential growth described by $A_{n+1}=\mu_{1} A_{n}$, where $\mu_{1} \simeq 1.041$ is the growth rate from linear stability calculations. Initially the correction to the linear growth rate is given by $\mu_{1}-\alpha_{11} A_{n}^{2}$. Using the procedure described by Henderson \& Barkley (1996), the coefficient of this term may be estimated directly from the computational data as $\alpha_{11} \simeq-0.116$. Negative $\alpha_{11}$ indicates that the instability is subcritical.

Because the bifurcation to mode $\mathrm{A}$ is subcritical, the first two terms in (3.3) are insufficient for determining the limiting amplitude. Assuming $\alpha_{12}>0$, the lowest-order amplitude equation for the bifurcation to mode $\mathrm{A}$ becomes

$$
A_{n+1}=\left(\mu_{1}-\alpha_{11} A_{n}^{2}-\alpha_{12} A_{n}^{4}\right) A_{n} .
$$

Substituting $\mu_{1}=1+\mu_{1}^{\prime} \epsilon$, the equilibrium solutions to this equation for small $\epsilon$ are

$$
|A|^{2}=\frac{\left|\alpha_{11}\right|}{2 \alpha_{12}} \pm\left(\frac{\alpha_{11}^{2}}{4 \alpha_{12}^{2}}+\frac{\mu_{1}^{\prime} \epsilon}{\alpha_{12}}\right)^{1 / 2} .
$$

These amplitudes are shown as a bifurcation diagram in the inset to figure 5. Solid lines in the bifurcation diagram indicate stable states and dashed lines indicate unstable states. This diagram is necessarily schematic because the data do not permit a reliable estimate of the coefficient $\alpha_{12}$. However, its value is clearly positive and 


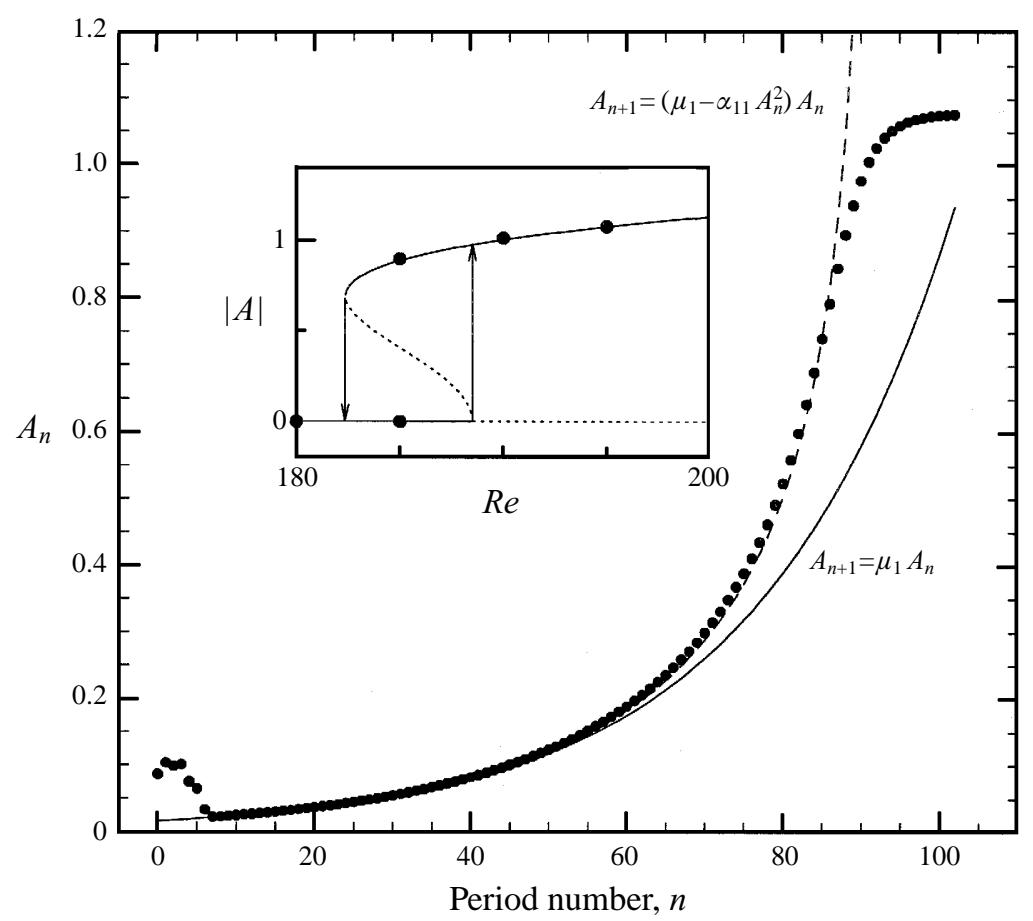

FIGURE 5. Nonlinear growth of a three-dimensional perturbation to the wake near the secondary instability threshold at $R e_{2} \simeq 190, \lambda_{2}=3.96 d$ : $\bullet$, values of the amplitude $A_{n}$ evaluated from simulations of the full Navier-Stokes equations at $R e=195$; curves show predictions from equation (3.5) truncated at first and third order with $\mu_{1}=1.041$ and $\alpha_{11}=-0.116$. The inset shows a bifurcation diagram along with simulation results at nearby parameter values.

approximately equal to $\alpha_{12} \approx 0.122$. Additional calculations were performed at nearby parameter values (see figure 1) to validate the predictions of finite-amplitude states and the subcritical nature of the instability. At $R e=190$ the flow was also found to be subcritical with $\alpha_{11} \simeq-0.116$. Below the critical point at $R e=185$ the flow was found to be bi-stable: initial conditions corresponding to $A_{0}=0.1$ decayed back to zero, while initial conditions corresponding to $A_{0}=0.915$ evolved to a saturated three-dimensional state with $A_{\infty}=0.897$. At $R e=180$ the flow was found to be stable to finite-amplitude perturbations, providing a lower bound on the turning point and confirming that the bifurcation diagram in figure 5 is approximately correct. There is clearly good agreement between the predictions of finite-amplitude states from equation (3.5) and full nonlinear calculations for Reynolds numbers near the critical point.

In addition to the finite-amplitude states that evolve from the linear instability, it is also interesting to look at how this bifurcation affects the global oscillation frequency of the wake. Although values of $t_{n}$ are not evenly spaced in time, the phase of the oscillation corresponding to zero lift provides a reference for defining the shedding period: $C_{L}\left(t_{n}\right) \equiv 0$. The distance between points $t_{n}$ changes smoothly during the transition to the new limit cycle and there is a well-defined instantaneous shedding period, $T_{n} \equiv t_{n+1}-t_{n}$. We define the instantaneous shedding frequency as $f_{n} \equiv T_{n}^{-1}$. Simulations near the critical point suggest the following hypothesis: the global oscillation frequency is determined by the the primary instability mode $\phi_{0}$, 


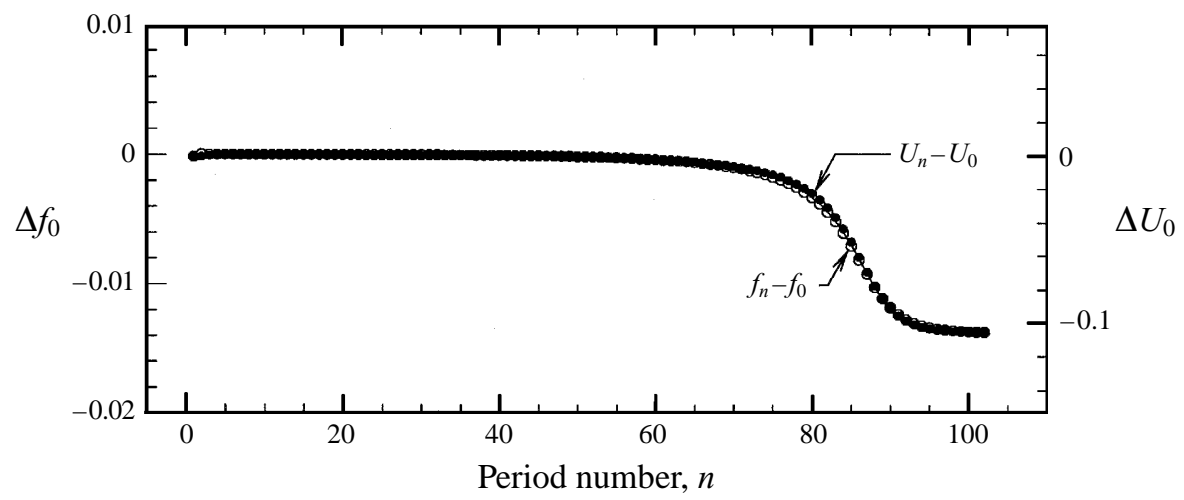

FIGURE 6. Nonlinear frequency shift due to a perturbation at $R e=195, \lambda=\lambda_{2}=3.96 d$ (same time series as figure 5): $\circ$, shift in global oscillation frequency (scale on the left); $\bullet$, shift in amplitude of the primary instability mode (scale on the right); the solid line is computed from equation (3.6) with parameter values $\gamma_{01}=0.01005, \gamma_{02}=0.00740$, and $\gamma_{03}=-0.00517$. The data verify that the global oscillation frequency follows the amplitude of the primary instability and explains the drop to the lower curve in figure 3.

and the oscillation of $\phi_{1}$ remains locked to this mode. $\dagger$ Since changes in $\phi_{0}$ are largely characterized by changes in its amplitude, we can write $f_{n}=F\left(U_{n}\right)$. Near the secondary instability threshold $F\left(U_{n}\right)$ can be expanded in a Taylor series about its two-dimensional value, $f_{0}=F\left(U_{0}\right)$ :

$$
\begin{aligned}
f_{n}=F\left(U_{n}\right) & \approx F\left(U_{0}\right)+\frac{\mathrm{d} F}{\mathrm{~d} U}\left(U_{n}-U_{0}\right) \\
& =f_{0}-\text { const. } \times \alpha_{01} A_{n}^{2} \\
& =f_{0}-\gamma_{01} A_{n}^{2} .
\end{aligned}
$$

Higher-order corrections will involve an expansion in even powers of $A_{n}$, and the frequency shift can be written in the same form as equation (3.2):

$$
f_{n}=f_{0}-\sum_{j=1}^{\infty} \gamma_{0 j} A_{n}^{2 j} .
$$

Like the coefficients $\alpha_{0 j}$, the values of $\gamma_{0 j}$ are $\epsilon$-dependent with variations of $O\left(\epsilon^{2}\right)$ or smaller; they will also be assumed constant for $|\epsilon| \ll 1$.

The computed shift in global oscillation frequency $f_{n}$ due to the growth and saturation of the mode A instability is shown in figure 6, with data which correspond to the same time series presented in figure 5. The change in amplitude of the primary instability mode $U_{n}$ is overlayed with the frequency data, showing that the two are indeed of the same form. Equation (3.6) truncated at $j=3$ with the given parameter values reproduces the computed frequency shift almost exactly. This change in frequency during the bifurcation from the two-dimensional state to mode A vortex shedding accounts for the drop to the lower curve in figure 3. Because the bifurcation to mode $\mathrm{A}$ is subcritical, there is a discontinuous jump to the three-dimensional state with a correspondingly large change in the amplitude of the primary and secondary

$\dagger$ One can also think of $\phi_{1}$ as a three-dimensional structure that wraps around the two-dimensional structure of $\phi_{0}$. It does not introduce a new time scale, but it can shift the time scale of the primary instability. 
instability modes. Since $f_{n}-f_{0} \propto A_{n}^{2}$, this jump is reflected by a large drop in the global oscillation frequency of the wake.

Perhaps the most interesting characterization of the state that exists after transition is in terms of the three-dimensional structure of the flow. Figure 7 shows a visualization of the fully saturated mode A state that evolves at $R e=195$, just beyond the secondary instability threshold. Figure 7(a) shows isosurfaces of the three-dimensional vorticity field, $\boldsymbol{\xi} \equiv \nabla \times \boldsymbol{u}$. The spatiotemporal symmetry of mode A (Barkley \& Henderson 1996, equation (3.3)) produces a staggered array of streamwise vortices that alternate in sign from period to period at a given spanwise location. Pattern formation in the system is shown using normalized grey-scale images of the streamwise and normal velocity components along the midplane of the wake. At saturation mode A produces a significant distortion of the Kármán vortex shedding pattern that gradually decreases in amplitude with distance downstream from the cylinder. In the discrete-time system this is a stationary spatial pattern centred around the critical mode wavelength $\lambda=\lambda_{2}=3.96 d$. The saturated state is perfectly time-periodic and the image shown is repeated exactly from period to period. The pattern does not 'wander' along the span or exhibit any other type of irregular behaviour. This spatially periodic flow pattern represents an idealization of mode A observed in experiments near the secondary instability threshold (see e.g. Williamson 1988, 1991).

\subsection{Bifurcation to mode $B: R e \simeq 260, L=0.822 d$}

Next we consider perturbations to the wake near the short-wavelength instability threshold at $R e=265, \lambda=\lambda_{2}^{\prime}=0.822 d$. The procedure is exactly the same, and the evolution of the flow was also computed using $M=16$ modes. Mode A does not appear owing to the restricted size of the domain. Figure 8 (see p. 86) shows the results from this calculation. Starting from a small perturbation, the instability saturates after $O(50)$ shedding periods. The linear growth rate of the perturbation is $\mu_{1} \simeq 1.16$. However, in this case the amplitude drops below the exponential growth curve near saturation. The estimated value of the Landau constant for this bifurcation is $\alpha_{11} \simeq 3$.92. Large positive $\alpha_{11}$ indicates that the instability is strongly supercritical.

Only two terms are needed to determine the limiting amplitude of a supercritical instability. The lowest-order amplitude equation for the bifurcation to mode B is therefore

$$
B_{n+1}=\left(\mu_{1}-\alpha_{11} B_{n}^{2}\right) B_{n} .
$$

For $\epsilon<0$ the flow always decays back to a two-dimensional state $(B=0)$, while for $\epsilon>0$ there are finite-amplitude states given by

$$
|B|^{2}=\mu_{1}^{\prime} \epsilon / \alpha_{11} .
$$

These amplitudes are shown as a bifurcation diagram in the inset to figure 8 . The supercritical nature of the bifurcation to mode $\mathrm{B}$ was verified by performing simulations at nearby parameter values near the tip of the mode B instability region (see figure 1). Simulations at $R e=260$ (just above the critical point at $R e_{2}^{\prime}=259$ ) and $R e=270$ both evolved to three-dimensional states that lie near the predicted limiting amplitude. Calculations below the critical point at $R e=255$ decayed back to a two-dimensional state. Small perturbations at $\lambda=\lambda_{2}^{\prime}$ and smaller are stable for $\operatorname{Re}<\operatorname{Re}_{2}^{\prime}$.

One would not expect a large change in global oscillation frequency near the onset of mode B for the following reason. A supercritical bifurcation represents a continuous transition from the two-dimensional state. Any coupling to the primary instability 
(a)

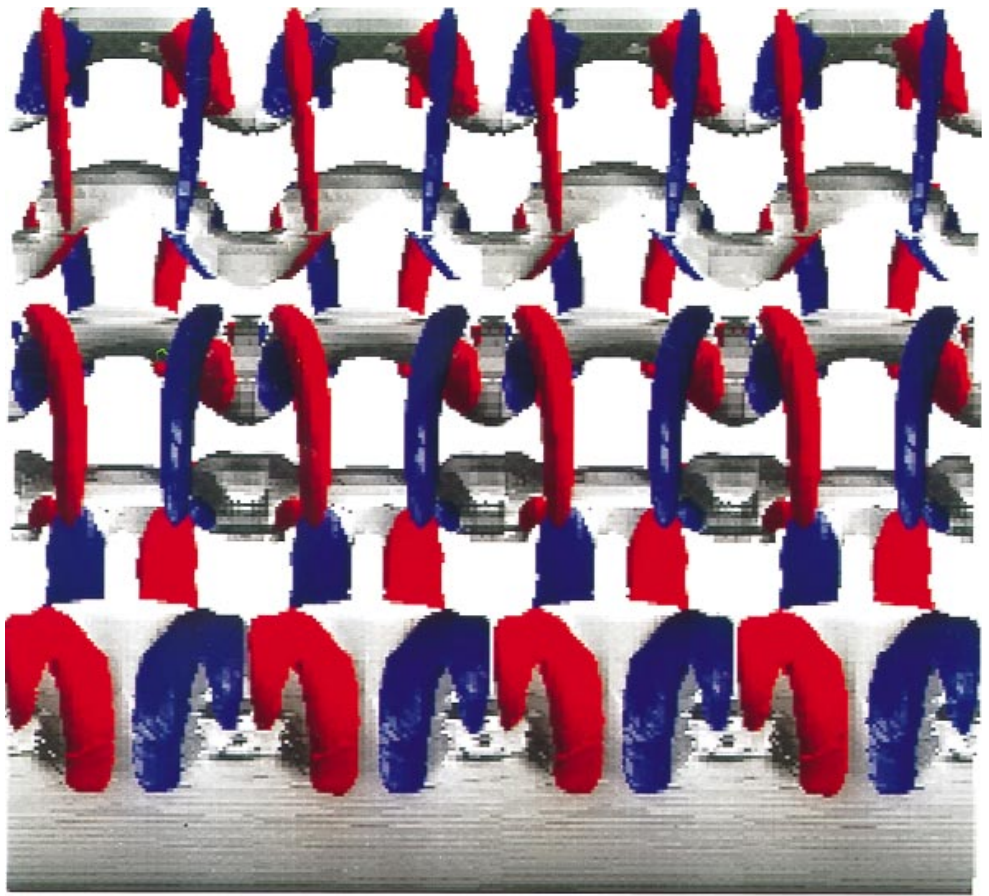

$\xi_{x}=+0.75$ (red), -0.75 (blue); $\left|\xi_{z}\right|=1$ (silver)

FiguRE 7. (a). For caption see facing page.

(a)

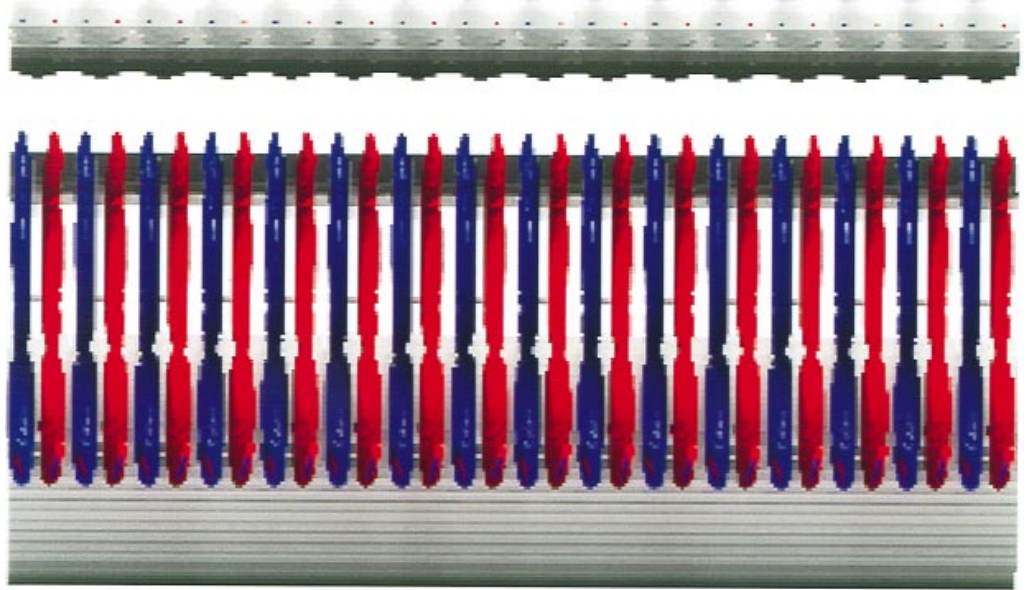

$\xi_{x}=+0.5$ (red), -0.5 (blue); $\left|\xi_{z}\right|=1$ (silver)

FiguRE 9. (a). For caption see facing page.

mode is weak near onset because the bifurcating mode saturates at low amplitude $|B|^{2} \sim \epsilon$. For $0<\epsilon \ll 1$ both the global oscillation frequency and amplitude of the primary instability mode remain constant to $O(\epsilon)$ during the transition from the two-dimensional state to mode B. Although there will be a measurable change for sufficiently large $\epsilon>0$, the computations do not indicate any significant change in 
(b)

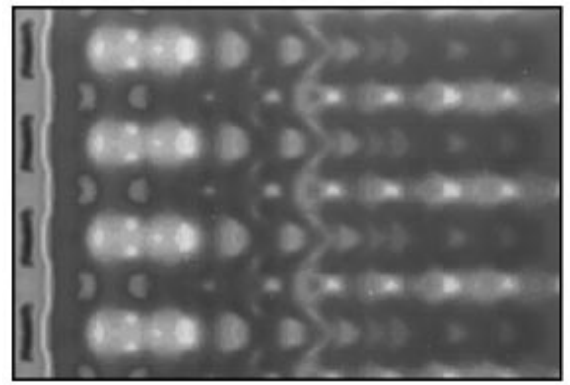

$u(x, y=0, z, t)$

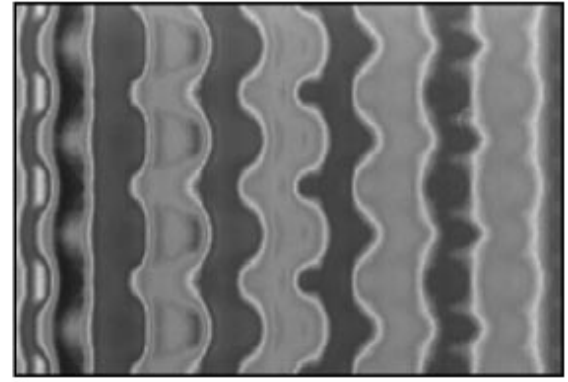

$v(x, y=0, z, t)$

FIGURE 7. Visualization of mode A after transition at $R e=195$ : (a) isosurfaces of the three-dimensional vorticity field; $(b)$ spatial pattern formation in the velocity field, centred around a spanwise wavelength of $\lambda=\lambda_{2}=3.96 d$. The image plane corresponds to $x / d=(0,24)$ and $z / d=(0,15.84)$, with a somewhat shorter downstream extent in image $(a)$.

(b)

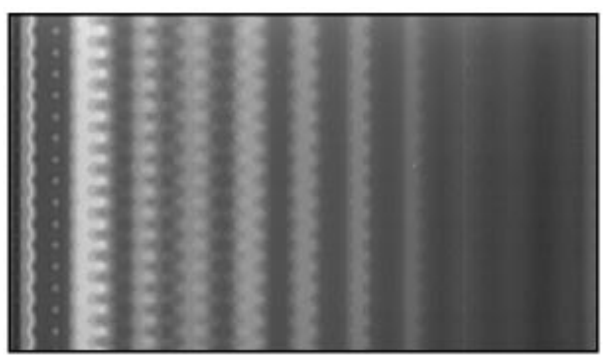

$u(x, y=0, z, t)$

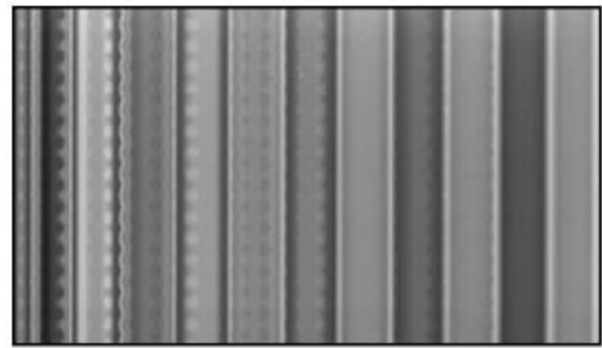

$v(x, y=0, z, t)$

FIGURE 9. Visualization of mode $\mathrm{B}$ after transition at $R e=265$ : (a) isosurfaces of the three-dimensional vorticity field; $(b)$ spatial pattern formation in the velocity field, centred around a spanwise wavelength of $\lambda=\lambda_{2}^{\prime}=0.822 d$. This flow pattern cannot be observed directly in experiments because it is unstable to longer-wavelength modes in the A-band. The image plane corresponds to $x / d=(0,24)$ and $z / d=(0,13.12)$, with a somewhat shorter downstream extent in image $(a)$.

shedding frequency for $R e \approx R e_{2}^{\prime}$ when the three-dimensional flow is restricted to pure mode B.

Figure $9(a)$ shows the three-dimensional structure of the flow that evolves at $R e=265$ as mode $\mathrm{B}$ develops in the wake. The spatiotemporal symmetry of mode $\mathrm{B}$ (Barkley \& Henderson 1996, equation (3.4)) produces an inline array of streamwise vortices that have the same sign from period to period at a given spanwise location. This can also be interpreted as the symmetry of mode A plus a shift of the threedimensional structure by half a wavelength after a time $T / 2$. Figure $9(b)$ shows spatial pattern formation in the velocity field. To emphasize the relationship between the long- and short-wavelength instabilities, this and subsequent flow visualization in figures 11 and 13 are shown to the same scale. The nonlinear saturated state produces only a slight distortion of the basic two-dimensional flow with an amplitude that decays rapidly with distance downstream of the cylinder. At moderate distances downstream there is no evidence of the mode B instability and the wake returns to a two-dimensional state. As in the case of mode A, the saturated flow is time-periodic 


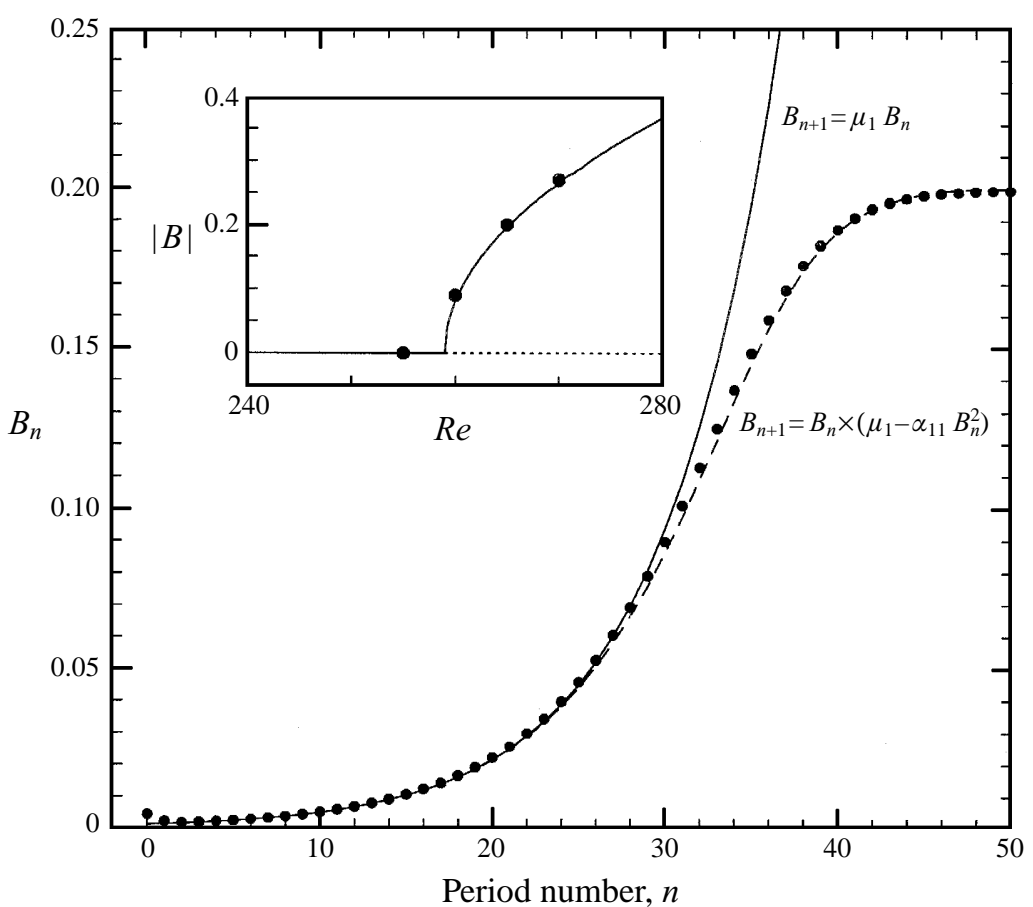

FIGURE 8. Nonlinear growth of a three-dimensional perturbation to the wake near the secondary instability threshold at $R e_{2}^{\prime} \simeq 260, \lambda_{2}^{\prime}=0.822 d$ : $\bullet$, values of the amplitude $B_{n}$ evaluated from simulations of the full Navier-Stokes equations at $R e=265$; curves show predictions from equation (3.7) truncated at first and third order with $\mu_{1}=1.16$ and $\alpha_{11}=3.92$. The inset shows a bifurcation diagram along with simulation results at nearby parameter values.

and the spatial pattern is stationary. Although the flow pattern shown in figure 9 has some qualitative similarities with mode B observed in experiment, this 'pure' state cannot be observed directly in the laboratory because the wake is absolutely unstable to mode $\mathrm{A}$ in this range of Reynolds number. We explore this issue in detail in the following section.

\subsection{Spatiotemporal chaos: $R e \simeq 260, L \rightarrow \infty$}

We now turn our attention away from the critical points to look at the dynamics of the wake in a sequence of systems with fixed control parameter Re and increasing spanwise dimension $L$. The sequence is computed at $R e=265$ where both Aand B-type instabilities are self-excited (see figure 1). Note that the most-amplified mode A wavelength is not constant but decreases with Reynolds number (Barkley $\&$ Henderson 1996). At $R e=265$ the ratio of the critical mode $\mathrm{B}$ wavelength to the fastest growing mode A instability falls close to a power of $\left.2\left(\lambda \simeq 3.288 d=4 \times \lambda_{2}^{\prime}\right)\right)$. Since this is not true at other values of the Reynolds number, $R e=265$ is a unique choice for computing the sequence $2^{n} L$ because the discrete spectrum includes the largest eigenvalue in both the mode $\mathrm{A}$ and mode $\mathrm{B}$ instability regions. The results of the previous section constitute the first element of this sequence, i.e. the development of the flow in a system where the spanwise dimension is equal to the critical mode B wavelength, $L=\lambda_{2}^{\prime}=0.822 d$. Subsequent calculations are performed at four times and sixteen times this length scale.

In analysing the complex flows that develop in these 'large' systems we will try to 
synthesize the previous results for bifurcations to pure states, but the approach will be slightly different. For moderate $L$ there is a finite number of global modes that participate in the dynamics. These modes are determined by the discrete wavenumbers that fall into a region of instability in figure 1. An amplitude equation describing the evolution of a single bifurcating mode near threshold does not directly apply. To interpret the process of nonlinear growth and saturation in a qualitative way we will rely on flow visualization. In order to quantify how the three-dimensional flow develops we can follow the amount of kinetic energy $E_{q}(t)$ carried by each Fourier mode during transition. The instantaneous kinetic energy at a given wavenumber may be computed approximately as

$$
E_{q}(t)=\frac{1}{2} \rho \int_{\Omega}\left|\hat{\boldsymbol{u}}_{q}\right|^{2} \mathrm{~d} \Omega .
$$

The time-averaged kinetic energy over some interval $\left(t_{0}, t_{0}+T\right)$ is given by

$$
\left\langle E_{q}\right\rangle=\frac{1}{T} \int_{t_{0}}^{t_{0}+T} E_{q}(t) \mathrm{d} t .
$$

We refer to the variation of $\left\langle E_{q}\right\rangle$ with $q$ as the spanwise energy spectrum. The spectrum is normalized so that $\left\langle E_{0}\right\rangle=1$. Like the global mode amplitude defined in (3.4), the numerical values of $E_{q}(t)$ also depend on the region $\Omega$ where the integrals in (3.8) are evaluated. There is a direct link between the energy spectrum and flow visualization: a 'spike' in the spanwise energy spectrum corresponds to a spatial structure with a well-defined spanwise wavelength. This provides an analogy to the link between global mode amplitude and pattern formation near the critical points.

\subsubsection{Quasi-periodic behaviour, $L=3.288 d$}

The first case we consider is a system with spanwise dimension four times larger than the critical mode B wavelength, $L=4 \times 0.822 d=3.288 d$. The evolution of this flow was also computed using $M=16$ modes since the highest wavenumbers still lie within the viscous-dominated regime. The fundamental $\hat{\boldsymbol{u}}_{1}$ with wavelength $\lambda=3.288 d$ corresponds to the most-amplified mode of the wake at $R e=265$; the wavelength of the harmonic $\hat{\boldsymbol{u}}_{4}$ lies just inside the tip of the mode B instability region; all other wavelengths correspond to linearly stable modes. Since there are two self-excited instabilities in this system, we expect the dynamics to represent the interaction of three global modes: $\phi_{0} \propto \hat{\boldsymbol{u}}_{0}, \boldsymbol{\phi}_{1} \propto \hat{\boldsymbol{u}}_{1}$ (mode A), and $\phi_{2} \propto \hat{\boldsymbol{u}}_{4}$ (mode B). Note that the full three-dimensional representation of $\phi_{1}$ and $\phi_{2}$ involves a sum over different subsets of the Fourier modes $\hat{\boldsymbol{u}}_{q}$, some of which carry shared components of the global modes. Although the fundamental mode for $\phi_{2}$ is a fourth harmonic of $\phi_{1}$, the two global modes are decoupled due to the distinct spatiotemporal symmetry of modes $\mathrm{A}$ and $\mathrm{B}$.

We start by looking at the shift in global oscillation frequency during the transition to the three-dimensional state, shown in figure 10. Initially there is a large drop in frequency due to the growth and saturation of the mode A instability, followed by a fast transition to a different state where the flow oscillates over a relatively narrow band of seven frequencies. Unlike the bifurcations to pure mode A or pure mode B, the asymptotic state is quasi-periodic with an oscillation period much longer than that of the two-dimensional flow. In this quasi-periodic state the average shedding frequency rises above the saturated mode A value and at times returns almost exactly to the two-dimensional value $f_{0}$. There are small variations in the flow during each 


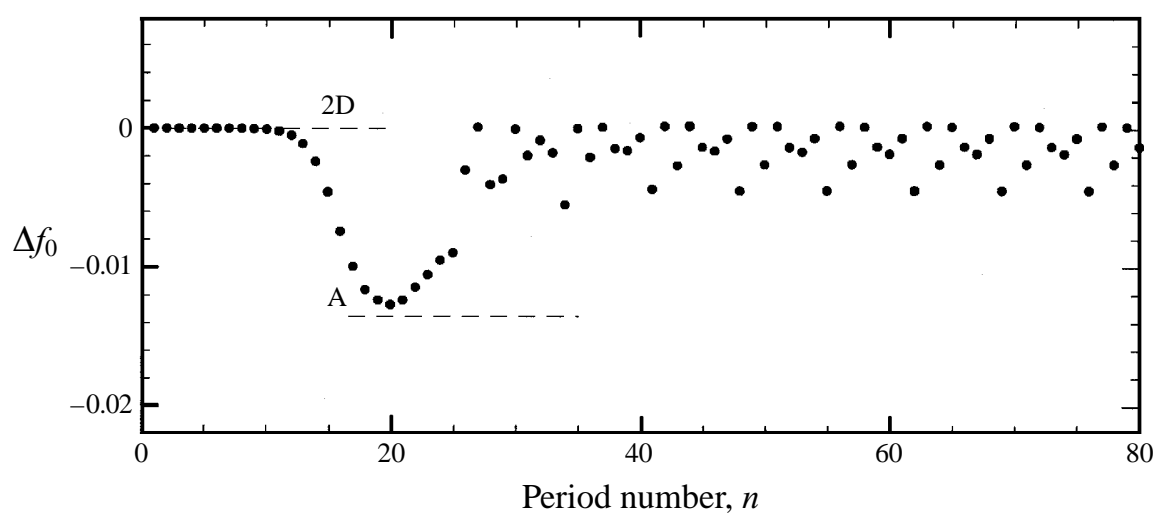

FIGURE 10. Shift in oscillation frequency during transition at $R e=265$ for calculations on the domain $L=3.288 d$. The asymptotic state is quasi-periodic and the wake oscillates within a group of seven frequencies.

of the intermediate shedding cycles, but no attempt was made to characterize the variations precisely.

These changes in oscillation frequency are linked to the spatial development of the flow. Figure 11 illustrates the structure of the wake as a series of patterns formed during transition. The entire sequence from initial perturbation to complete saturation takes $O(50)$ shedding cycles. During this time the flow passes through two states. The first coherent pattern emerges just as $\phi_{1}$ (mode A) saturates. This is the same pattern associated with the critical mode at $R e_{2} \simeq 190$. All modes within the mode A instability region produce this same basic pattern. As the amplitude of $\boldsymbol{\phi}_{2}$ (mode B) grows the flow evolves to a second state identified by the appearance of the short-wavelength mode B pattern in the near wake, superimposed over the largerscale mode A pattern. Mode B appears later in time because it has a much smaller growth rate. The state that exists at long times is a mix of both instability modes with an amplitude that depends on distance downstream of the cylinder. Notice that the highly distorted mode A pattern in figure 11(a) becomes smoothed-out following the appearance of mode B in the near wake. The decrease in the amplitude of mode A coincides with the rise of the global oscillation frequency in figure 10. This is the link between figures 10 and 11: the growth of mode B causes a large reduction in the amplitude of mode A, driving the system back towards a more 'two-dimensional' state. We shall return to this point in $\S 4$.

\subsubsection{Chaotic behaviour, $L=13.152 d$}

Next we look at the development of the flow in a system where the spanwise dimension is sixteen times longer than the critical mode B wavelength, $L=16 \times$ $0.822 d=13.152 d$. The evolution of the flow was computed using a total of $M=64$ modes on the same computational domain. The initial perturbation to $\hat{\boldsymbol{u}}_{1}$ at the fundamental spanwise wavelength lies well outside the mode A instability region; the wavelengths of the first four harmonics $\hat{\boldsymbol{u}}_{2-5}$ lie within the mode A instability region; the wavelength of the harmonic $\hat{\boldsymbol{u}}_{16}$ lies just inside the tip of the mode $\mathrm{B}$ instability region (see figure 1). Note that the discrete-time linear growth rate of the first five modes is such that $\mu_{1}<1$ and $\mu_{2}<\mu_{3} \approx \mu_{5}<\mu_{4}$ (all greater than 1 and hence unstable). The system admits three incommensurate wavelengths $\lambda_{i}=\left(\frac{1}{3}, \frac{1}{4}, \frac{1}{5}\right) \times L$ in the mode $\mathrm{A}$ instability region, plus one subharmonic of the 

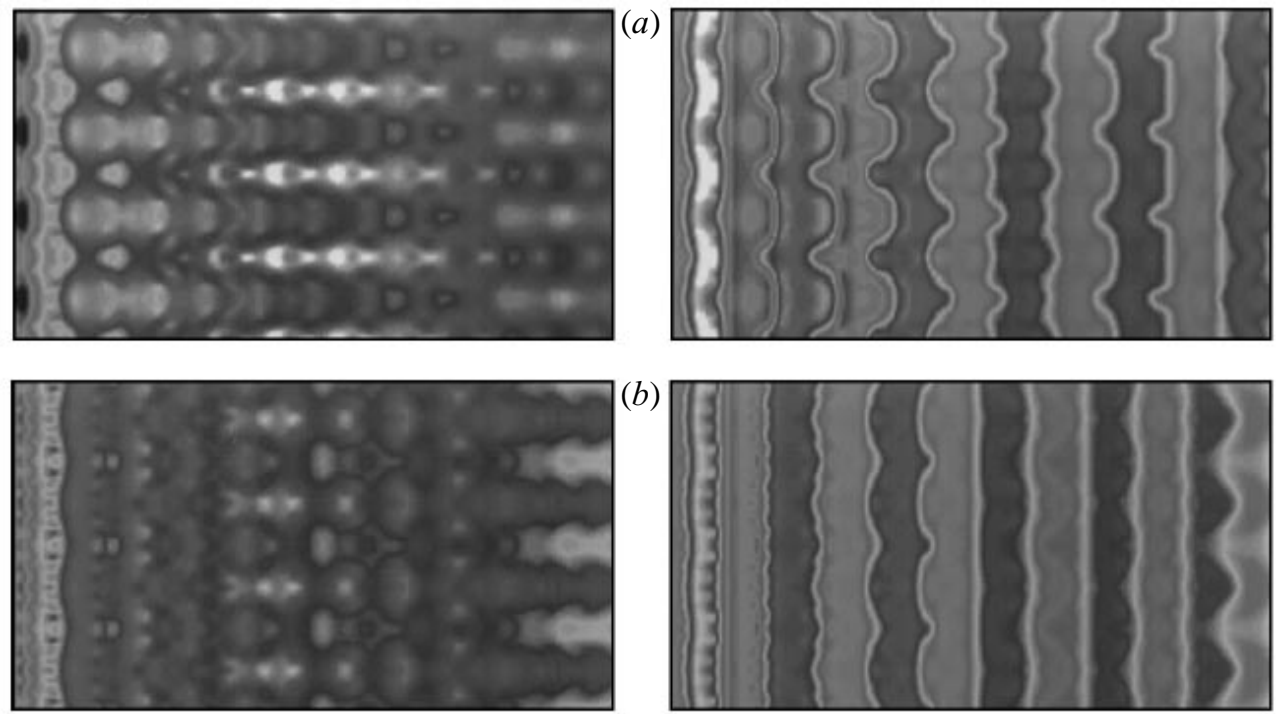

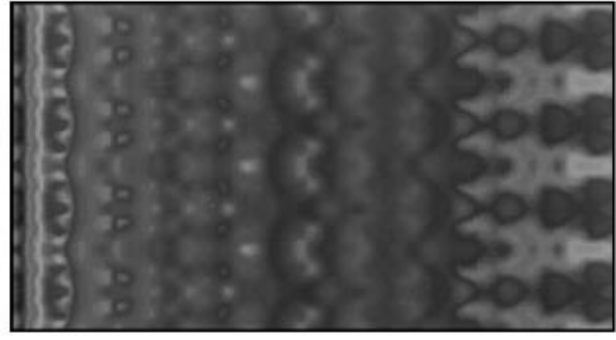

$u(x, y=0, z, t)$

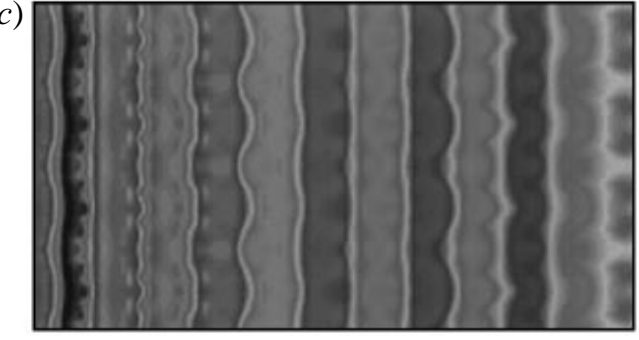

$v(x, y=0, z, t)$

FIgURE 11. Visualization of the three-dimensional flow that evolves from an initial perturbation at $R e=265, \lambda=3.288 d$ : (a) saturation of the most-amplified instability to give the mode A pattern; (b) the amplitude of mode A decreases as the mode B instability develops in the near wake; $(c)$ the asymptotic state is a mix of both modes. The spanwise dimension $L$ is only $1 / 4$ of the image shown.

fastest-growing mode A instability. We expect the dynamics to be described by the interaction of six global modes: the three modes of the previous section, plus three additional modes representing subdominant mode A instabilities. As before we can identify each global mode with its corresponding fundamental Fourier mode: $\phi_{0} \propto \hat{\boldsymbol{u}}_{0}$, $\boldsymbol{\phi}_{i} \propto \hat{\boldsymbol{u}}_{i+1}$ for $i=1 \ldots 4$ (mode A), and $\boldsymbol{\phi}_{5} \propto \hat{\boldsymbol{u}}_{16}$ (mode B).

As in the previous section we first look at the change in global oscillation frequency, shown in figure 12, as each of these instability modes develops in the wake. Initially the frequency drops as the most-amplified mode A instability grows, reaching a minimum value when the amplitude of this mode $\left(\phi_{3} \propto \hat{\boldsymbol{u}}_{4}\right)$ saturates. As before, the frequency again rises rapidly when mode B settles into the near wake. However, at long times the flow does not reach a periodic state. Instead it oscillates randomly within a narrow band of frequencies whose peak value is only slightly lower than that of the two-dimensional flow. For the remainder of this section we will concentrate on the bracketed region in figure 12 where the system makes a transition from regular to irregular dynamics.

The spatial development of the three-dimensional flow during this time is shown in figure 13. Similar to the transitional flow in the smaller system $L=3.288 d$, 


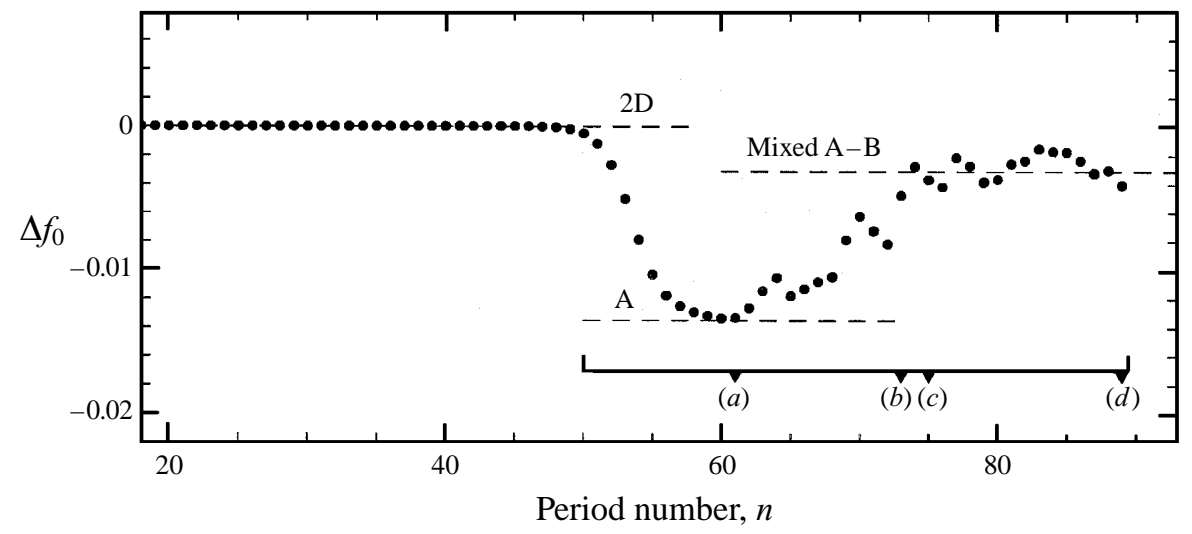

FIGURE 12. Shift in oscillation frequency during transition at $R e=265$ from calculations on the large domain $L=13.152 d$. The asymptotic flow is chaotic and oscillates within a narrow band of frequencies, slightly lower than that of the two-dimensional flow and centred around the level marked mixed $\mathrm{A}-\mathrm{B}$. Labels $(a-d)$ refer to the flow visualization in figure 13 .

the wake again passes through several states before reaching full saturation after $O(100)$ shedding periods. The first coherent pattern to emerge is identical to the one shown in figure 11(a) and corresponds to saturation of the most-amplified mode A instability $\left(\phi_{3} \propto \hat{\boldsymbol{u}}_{4}\right)$. Again the wake does not saturate around this state because subdominant modes continue to grow. The state following saturation of the leading mode is characterized by the appearance of irregular large-scale structures in the wake as shown in figure $13(b, c)$. These structures have remnants of mode $\mathrm{A}$ and are qualitatively similar to the 'vortex dislocations' reported by Williamson (1992). They are related to the growing amplitude of other self-excited mode A instabilities and lead to a breakdown of the regular mode A pattern. The state that exists at long times (figure 13(d)) does not exhibit any coherent large-scale structure. At long times the amplitude of all modes in the A-band equalizes to within an order of magnitude and the dynamics are chaotic. Mode B appears in the near wake in the same qualitative way as seen in figure 11(c): a small-scale pattern superimposed over the larger-scale structure of the wake.

The primary distinction between the structure of the flow in this calculation and the results for smaller systems is the development of these large-scale 'turbulent' structures due to the competition between multiple mode A instabilities. Figure 14 shows a closeup of the vorticity field just as mode A begins to break up. In the near wake there is a complex intertwining of the mode $\mathrm{A}$ and mode $\mathrm{B}$ instabilities. This figure also reveals an isolated mode A vortex structure at the core of the 'vortex dislocation' in figure $13(b)$. Otherwise, the instantaneous vorticity field is highly complex and difficult to analyse directly. It should be intuitively clear from both figures 13 and 14 that mode B dominates the near wake but quickly breaks up and is displaced by mode A slightly downstream. Also, compare the highly irregular state shown here with the pure mode B state shown in figure 9 , keeping in mind that both flow fields correspond to $R e=265$. Although mode B may appear to dominate the near wake, the effect of mode A disturbances clearly makes a fundamental difference to the character of the flow at this Reynolds number.

The transition from regular to chaotic dynamics is quantified in figure 15. This figure shows the instantaneous kinetic energy $E_{q}(t)$ for roughly half of the spectrum from the initial saturation of the leading mode A instability until the flow reaches 

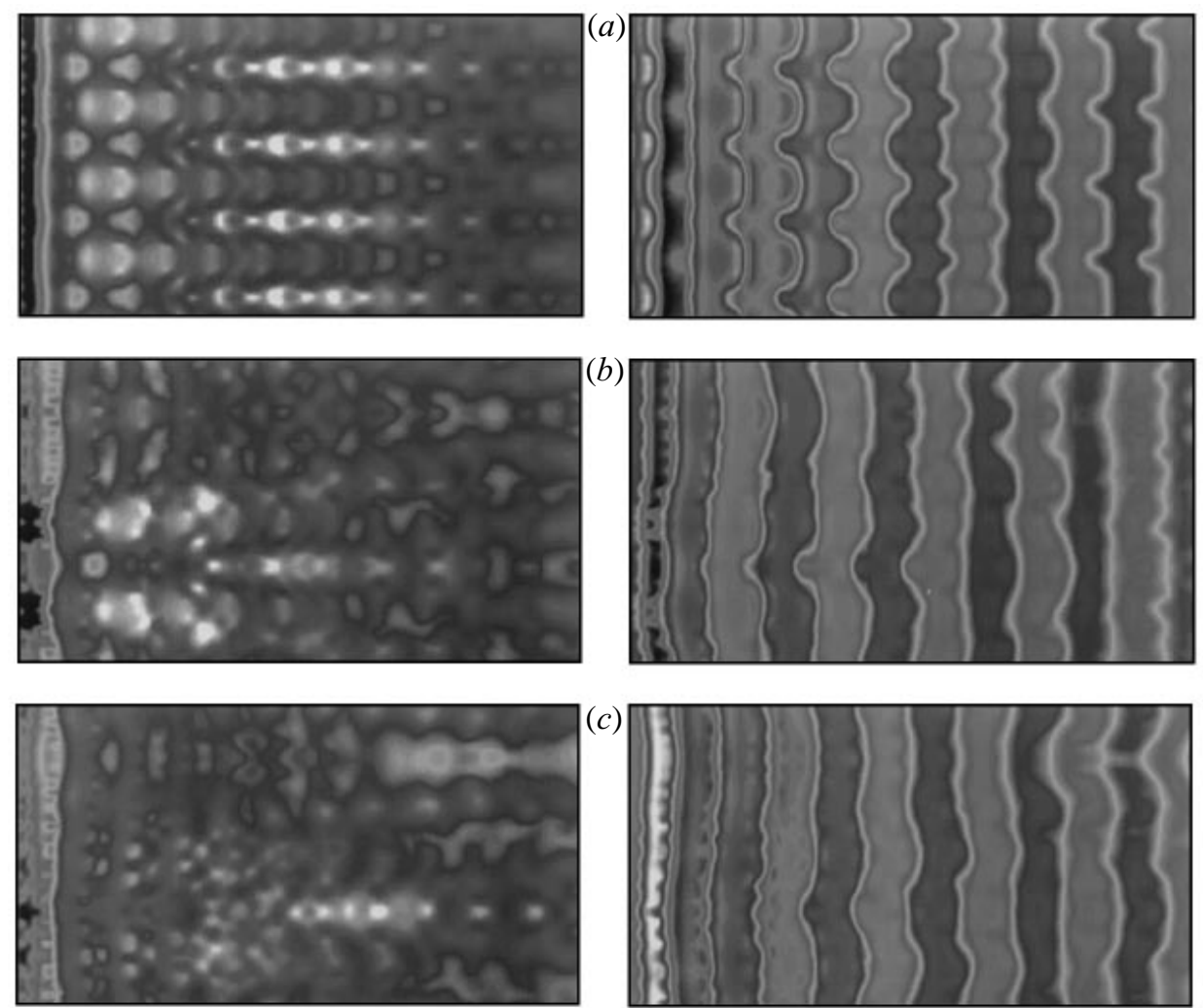

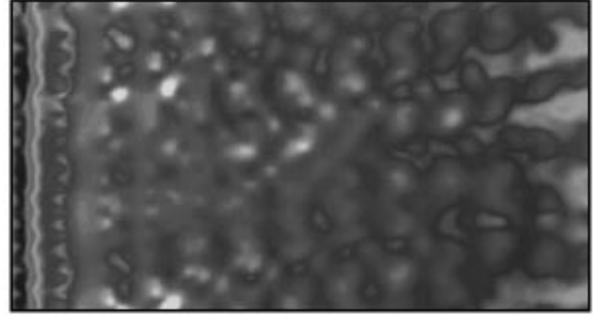

$u(x, y=0, z, t)$

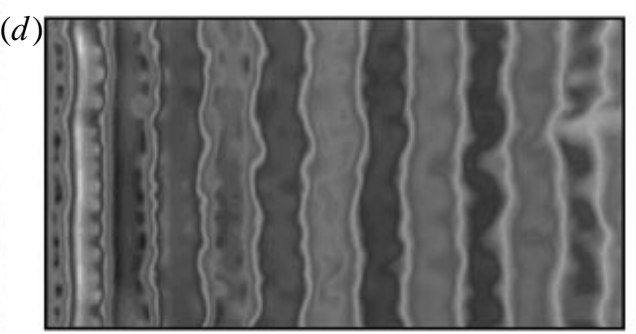

$v(x, y=0, z, t)$

FIGURE 13. Visualization of the three-dimensional flow that evolves from an initial perturbation at $R e=265, \lambda=13.152 d$ : (a) saturation of the most-amplified instability around a wavelength of $\lambda=3.288 d$ to give the mode A pattern; $(b, c)$ development of large-scale structure during the breakdown of the mode A pattern; $(d)$ asymptotic state with no discernible large-scale pattern. The images represent the full spanwise dimension of the system.

its asymptotic state. A set of labels is shown just above the $t$-axis so that the flow visualization in figures 13 and 14 can be compared directly with the spanwise energy spectrum in figure 15. Each bold trace indicates the amplitude of one Fourier mode identified as the fundamental mode for a self-excited global instability. The most important feature of the time-dependent energy spectrum is the observation that chaotic behaviour arises from the strong nonlinear interaction between the three leading mode $\mathrm{A}$ instabilities, beginning at $t-t_{0} \approx 290$.

We can clarify the interaction between secondary instability modes as follows. Whenever the flow admits two global modes $\boldsymbol{\phi}_{p}$ and $\boldsymbol{\phi}_{q}$ with fundamental wavenum- 


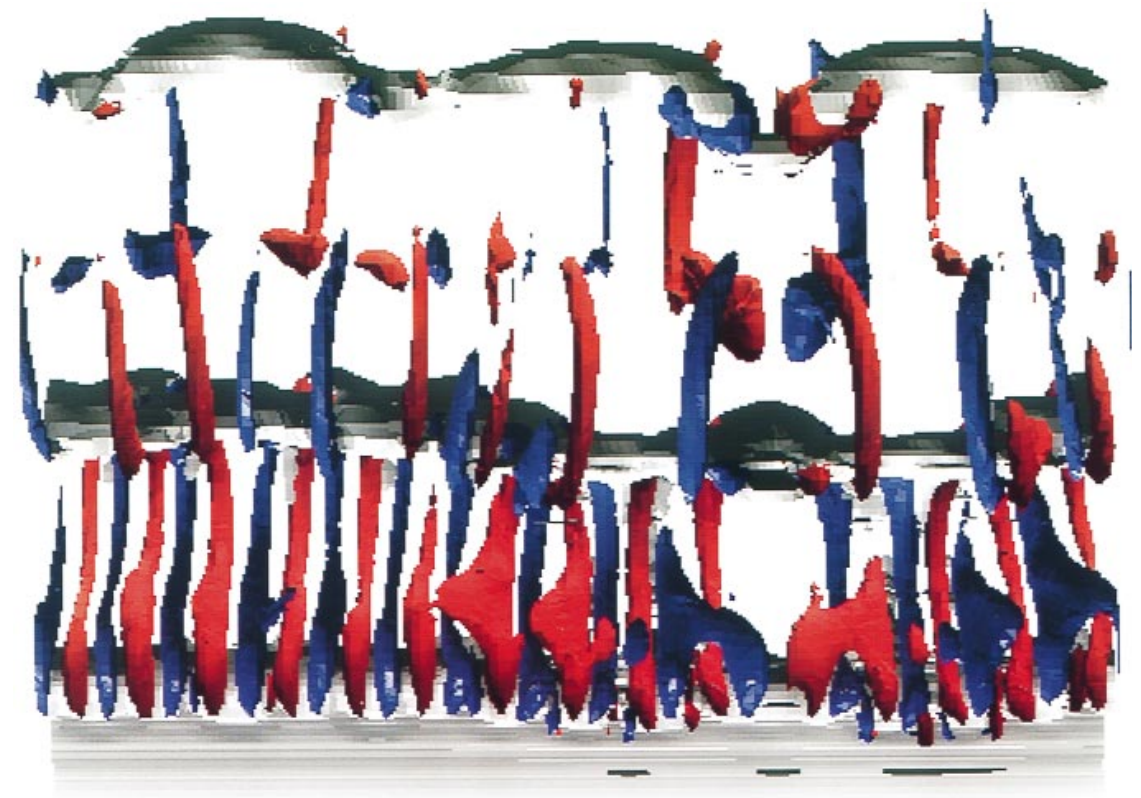

FigURE 14. Visualization of the three-dimensional vorticity field during transition at $R e=265$, just following the breakdown of mode A and subsequent transition to a mixed A-B state. Only $\xi_{z}=+1$ is plotted in order to reveal the streamwise vorticity in the near wake $\left(\xi_{x}= \pm 0.65\right)$, since otherwise it would be hidden by a sheet of negative spanwise vorticity shedding from the cylinder. Data for this image is taken from figure $13(b)$.

bers $\beta_{p}$ and $\beta_{q}$ (not integer multiples), there is a redistribution of energy through the nonlinear term in the Navier-Stokes equations:

$$
\boldsymbol{N}\left(\boldsymbol{\phi}_{p}+\boldsymbol{\phi}_{q}\right)=\left(\boldsymbol{\phi}_{p} \cdot \nabla\right) \boldsymbol{\phi}_{p}+\left(\boldsymbol{\phi}_{q} \cdot \nabla\right) \boldsymbol{\phi}_{q}+\left(\boldsymbol{\phi}_{p} \cdot \nabla\right) \boldsymbol{\phi}_{q}+\left(\boldsymbol{\phi}_{q} \cdot \nabla\right) \boldsymbol{\phi}_{p} .
$$

The first two terms on the right represent the self-interaction that links $\phi_{p}$ and $\phi_{q}$ back to the primary instability $(\beta=0)$ and to higher harmonics of their fundamental wavenumbers. This is the only type of nonlinear interaction in systems that admit a single secondary instability mode ( $\$ \$ 3.3$ and 3.4 ). Coupling between secondary instability modes is due to the last two terms on the right. These terms are responsible for both the forward cascade of energy to smaller scales,

$$
\boldsymbol{N}\left(\hat{\boldsymbol{\phi}}_{p}+\hat{\boldsymbol{\phi}}_{q}\right) \mathrm{e}^{\mathrm{i}\left(\beta_{p}+\beta_{q}\right)}+\boldsymbol{N}\left(\hat{\boldsymbol{\phi}}_{p}^{*}+\hat{\boldsymbol{\phi}}_{q}^{*}\right) \mathrm{e}^{-\mathrm{i}\left(\beta_{p}+\beta_{q}\right)},
$$

and the inverse cascade of energy to larger scales,

$$
\boldsymbol{N}\left(\hat{\boldsymbol{\phi}}_{p}+\hat{\boldsymbol{\phi}}_{q}^{*}\right) \mathrm{e}^{\mathrm{i}\left(\beta_{p}-\beta_{q}\right)}+\boldsymbol{N}\left(\hat{\boldsymbol{\phi}}_{p}^{*}+\hat{\boldsymbol{\phi}}_{q}\right) \mathrm{e}^{-\mathrm{i}\left(\beta_{p}-\beta_{q}\right)} .
$$

This interaction drives global modes other than $\boldsymbol{\phi}_{p}$ and $\boldsymbol{\phi}_{q}$. If there were no coupling between secondary instability modes we would expect the flow to saturate around the fastest-growing linear instability, producing a bifurcation to a pure three-dimensional state. Nonlinearity drives the amplitude of subdominant modes to a level far from their equilibrium values and the flow never reaches a regular state. In figure 15 this is led by the triad interaction between Fourier modes $\hat{\boldsymbol{u}}_{3}-\hat{\boldsymbol{u}}_{4}-\hat{\boldsymbol{u}}_{5}$ which transfers energy to both $\hat{\boldsymbol{u}}_{1}$ and $\hat{\boldsymbol{u}}_{2}$, causing the amplitude of higher-wavenumber modes to grow. The asymptotic state may be interpreted as a competition between multiple oscillators 


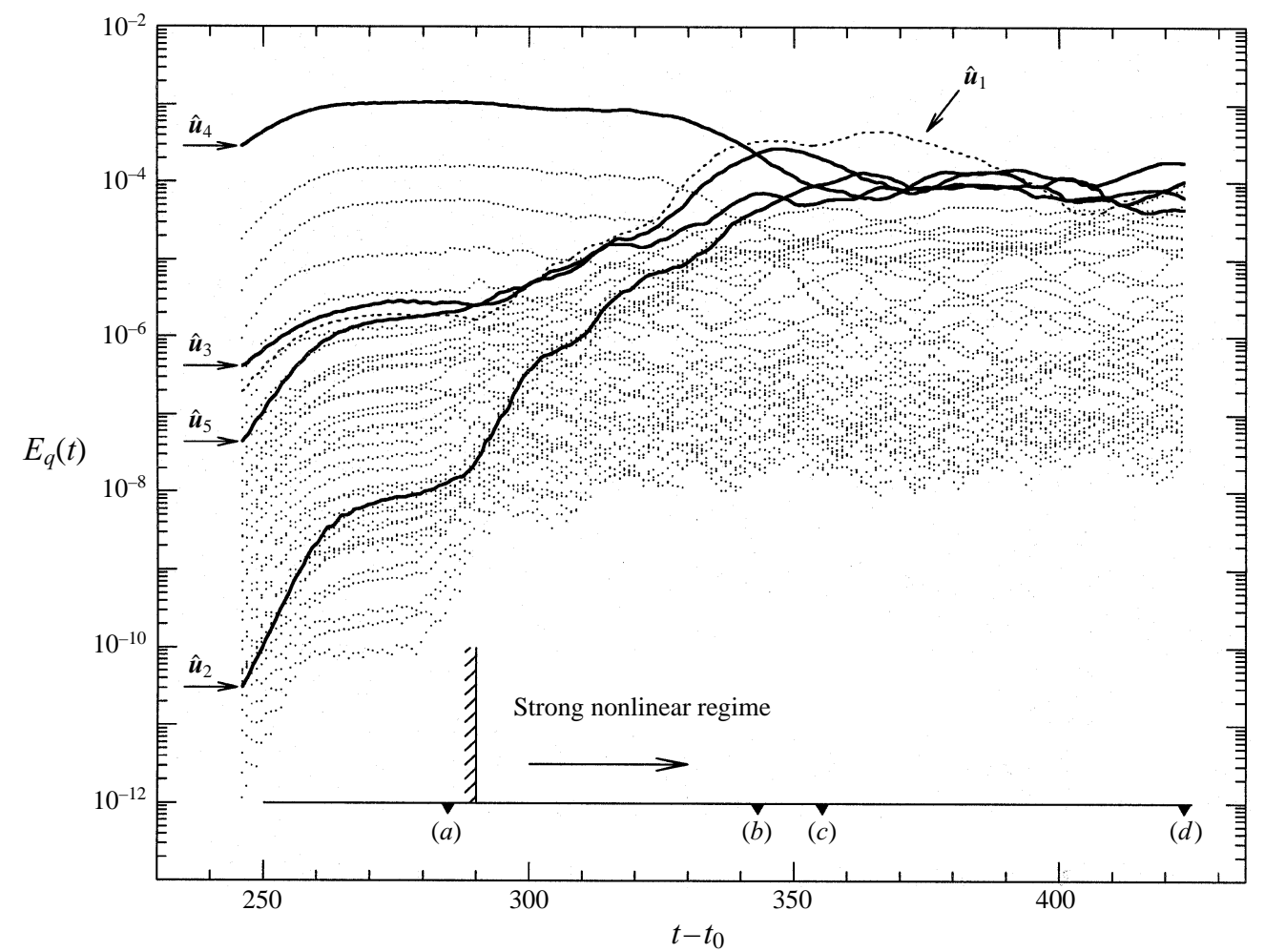

FIGURE 15. Spatiotemporal chaos in the wake at $R e=265$. Each trace corresponds to the energy of a three-dimensional mode with a given spanwise wavenumber (approximately half of the spectrum is shown). Bold traces correspond to the four modes that lie within the mode A instability region. At $t-t_{0} \approx 290$ the flow enters a strongly nonlinear regime where subdominant instability modes grow rapidly. At $t-t_{0} \approx 320$ large-scale turbulent structures appear in the wake, marking the breakdown of the regular mode A pattern and subsequent transition to the chaotic state that exists at long times. Labels $(a-d)$ refer to the flow visualization in figure 13.

represented by the self-excited global modes $\phi_{i}(x, t)$, each trying to reach a different equilibrium amplitude but driven off-peak by nonlinearity in the system.

The equilibrium amplitude that is reached by each mode in the fully coupled nonlinear system is characterized by the time-averaged form of the spanwise energy spectrum, shown in figure 16. The spanwise energy spectrum indicates the average distribution of energy in the three-dimensional wake. Figure 16 shows results from all calculations at $R e=265$. For the (time-periodic) flows in small systems the averaging is performed over the time scale for which the flow is periodic, while for the (nonperiodic) large system the averaging is performed over the time scale of figure 15 . Following the ideas of Hohenberg \& Shraiman (1989) we distinguish two basic length scales in the energy spectrum. The excitation length $l_{E}$ is the scale at which energy is extracted from the primary instability and injected into the three-dimensional flow. The dissipation length $l_{D}$ is the scale at which energy is dissipated by viscosity. In figure 16 we identify $l_{E}$ primarily with the mode A instability because the energy level in the mode $\mathrm{B}$ range is an order of magnitude lower. Based on the fact that mode $\mathrm{B}$ is strongly supercritical, we can estimate $l_{D}$ as being somewhat smaller than the critical mode B wavelength at $R e=265$. At higher Reynolds number the excitation 


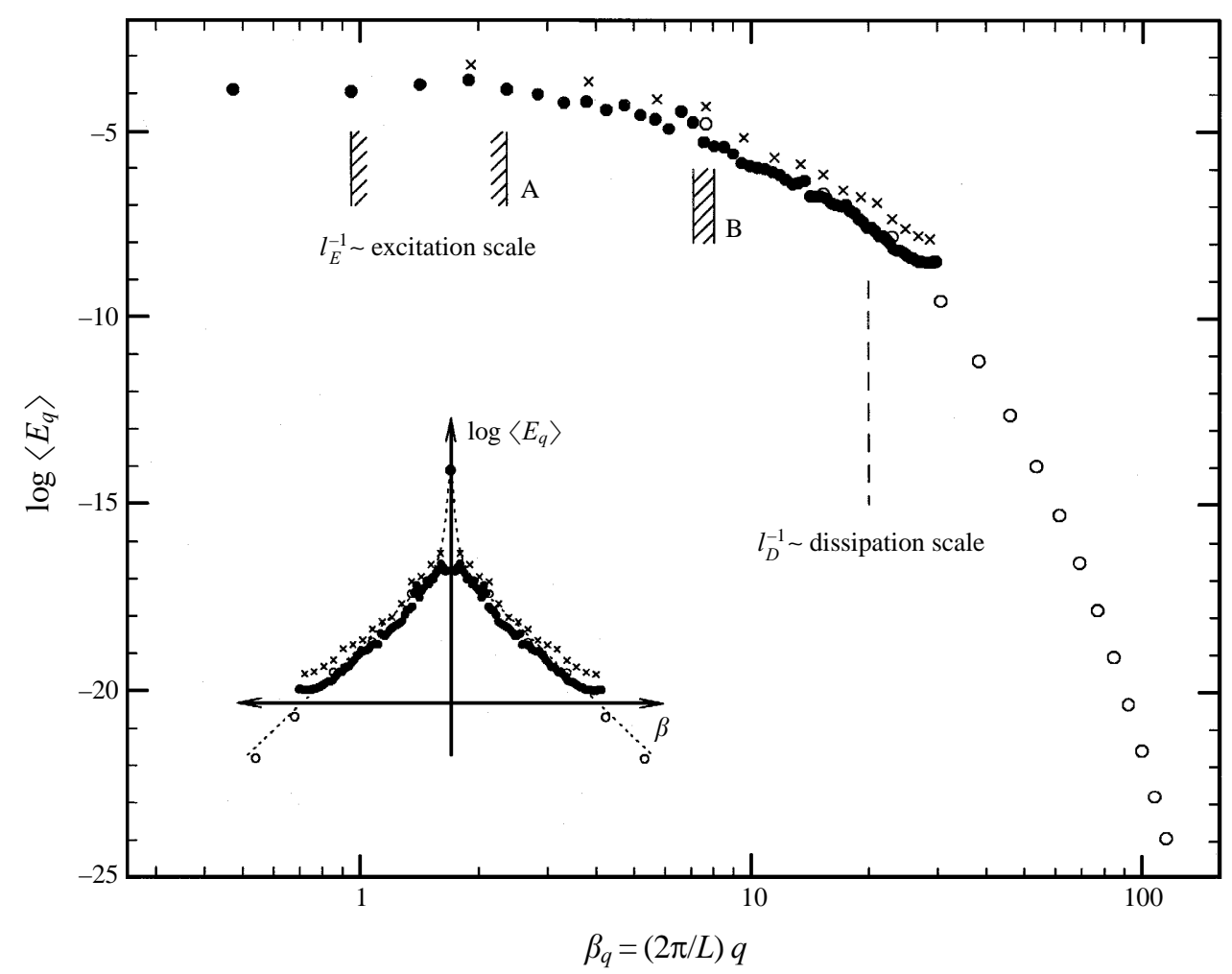

FIGURE 16. Long-time spanwise energy spectrum of the three-dimensional wake after transition at $R e=265$ for increasing system size: $\circ, L=0.822 d ; \times, L=3.288 d ; \bullet, L=13.152 d$. Shaded regions indicate the approximate wavenumber bands affected by the mode A and mode B instabilities. Also indicated are the excitation scale $l_{E}$ due to mode $\mathrm{A}$ and the approximate dissipation scale $l_{D}$ due to viscosity. The inset shows a portion of the data on a semilog scale to emphasize the connection with the primary instability at $\beta=0$.

scale should remain approximately constant while the dissipation scale decreases as $l_{D} \sim R e^{-1 / 2}$.

Low-wavenumber modes of the wake are linearly stable but essentially independent of viscosity (non-dissipative), and it is interesting that energy does not seem to fall off at low wavenumbers. While this result may seem odd at first, the reason is clear when the same data are plotted on a semilog scale as in the inset to figure 16 . The energy spectrum is driven by the primary instability at $\beta=0$, which in turn provides a sustained excitation through the secondary instability to a band of wavenumbers around $\beta \sim l_{E}^{-1}$. Energy is redistributed to all modes by nonlinearity until the flow reaches a saturated state, but energy is only dissipated at wavenumbers larger than $\beta \sim l_{D}^{-1}$. At long times there is a continuous distribution of energy at all scales in the three-dimensional wake, one of the essential features of a 'turbulent' flow.

We look at one other summary of the various calculations for $R e=265$ by plotting the surface pressure distribution as a function of system size $L$. The surface pressure coefficient is defined as $C_{p} \equiv\left(p-p_{\infty}\right) / \frac{1}{2} \rho u_{\infty}^{2}$, where $p$ is the mean static pressure on the surface of the cylinder. To convert from Cartesian to cylindrical coordinates we take $\theta=\tan ^{-1} y / x$ so that $\theta=0$ is the base of the cylinder and $\theta=\pi$ is the front stagnation point. Figure 17 compares the surface pressure from a two-dimensional simulation with the results of three-dimensional calculations for 


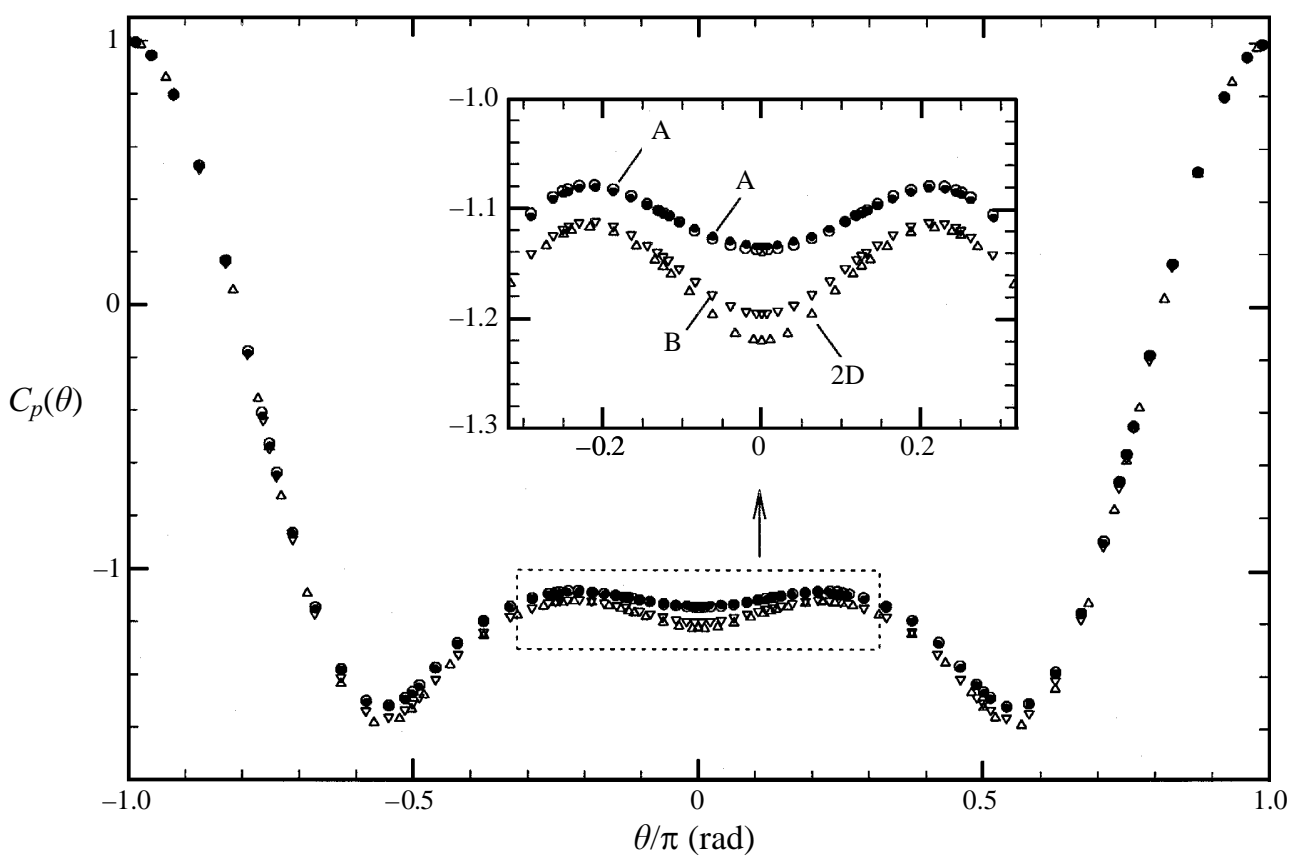

FIGURE 17. Surface pressure distribution for flow past a circular cylinder at $R e=265$. This sequence illustrates the effect of fixing the control parameter $R e$ and increasing the system size $L$, starting from a simulation of the two-dimensional wake: curve B $(L=0.822 d)$ only includes the short-wavelength instability; curve A $(L=3.288 d)$ includes the effect of a single long-wavelength instability mode; curve $\mathrm{A}^{*}(L=13.152 d)$ includes the effect of several long-wavelength modes and indicates that $C_{p}(\theta)$ has reached a level that is independent of system size $L$.

$R e=265$ and increasing system size $L$. Note that $C_{p}$ depends only on the component of pressure exerted by the mean flow $(\beta=0)$ since all other Fourier components have zero contribution when averaged along the span of the cylinder. Small-scale three-dimensionality alone $(L=0.822 d)$ results in a relatively small reduction in the magnitude of $C_{p}$ on the low-pressure side. This is because of the weak interaction between mode B and the primary instability near onset. The presence of mode A $(L=3.288 d)$ causes a much greater reduction in the magnitude of $C_{p}$. Calculations for the largest domain $(L=13.152 d)$ agree well with this curve and indicate that the computed surface pressure distribution has reached a level that is independent of the system size $L$.

Wake simulations at $R e=265$ with $L=13.152 d$ should represent all of the important qualities of the flow observed in larger experimental systems, excluding phenomena associated with end effects. In particular, larger systems at this Reynolds number will also exhibit spatiotemporal chaos due to the same nonlinear mechanism described above. Since this basic mechanism is in place for all $R e>R e_{2}$, we expect the calculations reported here for $R e=265$ to represent the dynamics of the flow in a qualitative way for lower Reynolds numbers as well. This was verified directly at $R e=220$ using a domain of spanwise dimension $L=14.78 d$ and $M=64$ modes. This domain size was specifically chosen to include the most-amplified mode of the wake at $R e=220(\lambda \simeq 3.695 d)$ and two subdominant mode $\mathrm{A}$ instabilities (see figure 1$)$. In this system the flow also evolves to a state of spatiotemporal chaos at long times. Note that the chaotic states at $R e=220$ and $R e=265$ both involve a minimum of 


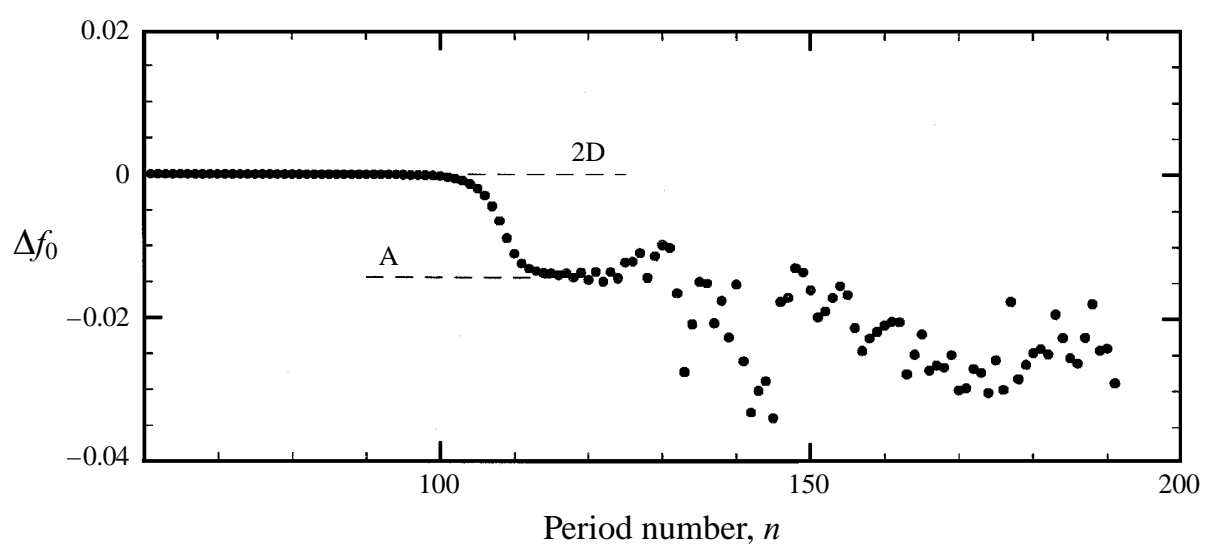

FIGURE 18. Shift in oscillation frequency during transition at $R e=220$ for calculations on the domain $L=14.78 d$. The asymptotic flow is chaotic, but at this Reynolds number the frequency remains low after transition because the amplitude of mode A remains large (see figure 3 ). The spatial development of the flow in time is similar to the sequence shown in figure 13.

three incommensurate wavelengths in the mode A instability region. The evolution of the flow at $R e=220$ is qualitatively similar to the wake structure shown in figure 13, including the appearance of large-scale disturbances just prior to the breakdown of the periodic mode A pattern. Figure 18 shows the shift in oscillation frequency and the onset of spatiotemporal chaos following saturation of the linear instability. At $R e=220$ the (nonlinear) excitation of mode B is relatively weak so the amplitude of mode A remains large. As a consequence the global oscillation frequency remains low in the asymptotic state. This is consistent with experimental observations in this range of Reynolds number and the computed frequency shift agrees well with the measured dominant frequency at $R e=220$ shown in figure 3 .

It is difficult to verify that the dynamics are chaotic at lower Reynolds number from direct numerical simulations of the full Navier-Stokes equations. Even the smallest system $L$ that includes a few wavelengths in the mode A instability region becomes very large as the width of the A-band shrinks near $R e_{2}$. For example, at $R e=190$ (just above the critical point at $R e_{2}=188.5$ ) the smallest system that includes three modes in the unstable mode A wavenumber band is $L \approx 75 d$, and $M \approx 300$ modes would be required to cover an appropriate range of scales. Intrinsically chaotic dynamics can still be inferred from the broad-band frequency spectra observed in experiments; we return to this point in $\S 4$.

\subsection{Simulations of the 'turbulent' wake: $R e=1000$}

The final set of calculations represent the flow far from the onset of the secondary instability, in the 'fully turbulent' regime at $R e=1000$. The most comprehensive calculations at this Reynolds number are performed on the large domain $M_{3}$ with a spanwise dimension of up to $L \approx 25 \mathrm{~d}$. The number of Fourier modes required to reach the dissipation scale in this system can be estimated as $\beta_{D}=(2 \pi / L) M \approx R e^{1 / 2}$, or $M \approx 128$ modes. At this resolution the calculations resolve the beginning of the dissipation range but not smaller scales, so the results must be considered somewhat qualitative. For example, there is a discrepancy of $10-20 \%$ between these calculations and experimental measurements of the mean drag coefficient and base pressure (see figures 4 and 21). However, the phenomena we wish to illustrate are related to the behaviour of the wake at large scales and these modes are fully resolved. As in the 
calculations at $R e=265$, we follow a sequence of fixed control parameter $R e$ and increasing system size from $L=2 \pi d \approx 6.283 d$ to $L=8 \pi d \approx 25.13 d$. The initial condition for the first calculation is a perturbation to the two-dimensional flow at $R e=1000$. Subsequent calculations are performed by taking the asymptotic flow on one domain and perturbing it at a lower wavenumber on a domain twice as large in the spanwise direction. The results illustrate some interesting pattern forming properties of the system at large Reynolds number and the effect that large-scale three-dimensionality in the wake has on the fluctuating forces and mean surface pressure distribution.

Figure 19 shows a visualization of the unsteady flow at $R e=1000$ on the largest domain $L=8 \pi d$. The sequence of images is spaced approximately two shedding cycles apart. The most striking feature is the spontaneous appearance of large-scale phase dislocations in the near wake. These are highly localized structures that differ qualitatively from the large-scale structures associated with the breakdown of mode A observed at lower Reynolds number. The dislocations appear intermittently in time and at random locations along the span. Various types of 'dislocations' or 'defects' are a common feature in many pattern-forming systems. In most fluid systems where dislocations have been studied experimentally, they are forced to occur by introducing some controlled non-uniformity to avoid the inherent randomness in time and space of naturally occurring dislocations.

Based on time traces of the spanwise energy spectrum (not shown), the phase dislocations in figure 19 seem to be caused by the rapid accumulation of energy in the low-wavenumber modes of the system. As the amplitude of these modes grows the shedding becomes out of phase over a distance equal to one-half their wavelength. If the amplitude becomes large enough, a dislocation occurs with a complex reconnection of vortices on either side. After the passage of a dislocation the wake is left in a 'winding state' that still satisfies the periodic boundary conditions along the span. Additional dislocations at later times destroy the winding state and return the wake to a state of nearly parallel shedding. This cycle of events occurs repeatedly but on a slow time scale with new dislocations forming after $O(10)$ shedding periods. The phenomenon is not understood at a fundamental level and is reported here only as an observation for this particular simulation. It is also unknown whether the irregular state depicted in figure 19 will continue indefinitely or settle down to a more regular but still 'turbulent' state after a long time.

The winding states observed in figure 19 are qualitatively similar to winding states reported by Leweke \& Provansal (1995) for the periodic wake of a ring at $R e=O(100)$. Blackburn \& Melbourne (1996) also show experimental evidence of winding states and phase dislocations in the wake of straight circular cylinders at Reynolds number as high as $R e=O\left(10^{5}\right)$. Similar structures are observed in other types of free shear flows as well. Browand \& Troutt $(1980,1985)$ showed that 'vortex defects' appear naturally during transition in the mixing layer at the boundary between cells of different frequency, or between adjacent cells with the same frequency but a different phase. Browand \& Prost-Domasky (1990) studied these structures in a controlled setting by acoustically forcing a shear layer at different frequencies along the span. In the wake simulations reported here the cylinder is perfectly uniform and there is no forcing. The only mechanism for generating dislocations is through the dynamics of large-scale structures in the wake.

Large-scale three-dimensionality in the wake also has a pronounced effect on the unsteady forces exerted on the cylinder. Figure 20 compares the fluctuating lift and drag for systems with increasing spanwise dimension $L$. These quantities are averaged 

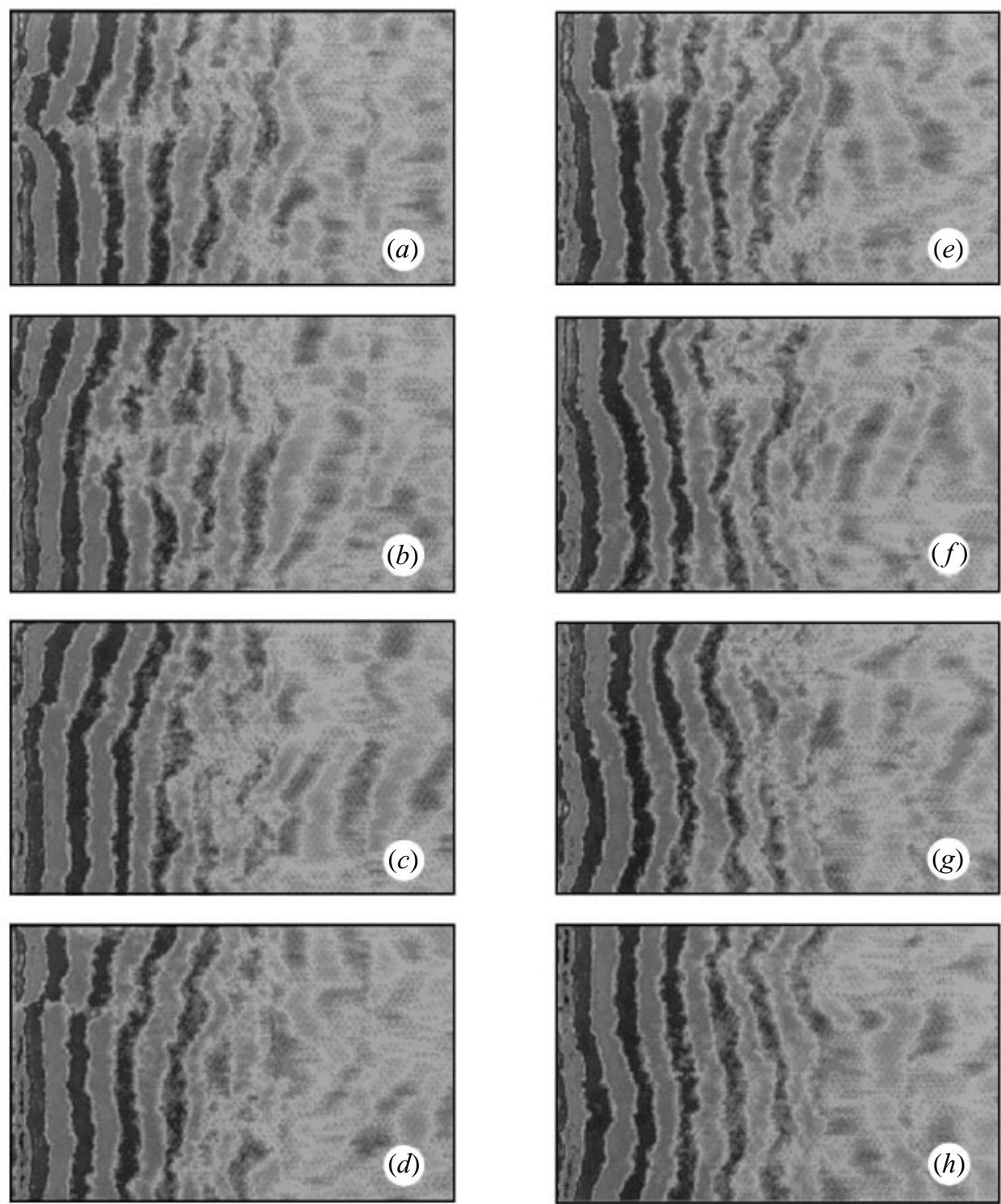

FiguRE 19. Visualization of the turbulent wake at $R e=1000$. The sequence of images $(a-h)$ shows contours of the normal velocity $v(x, y=0, z, t)$ at times spaced approximately two shedding cycles apart. The image plane corresponds to $x / d=(0,48)$ and $z / d=(0,25.13)$. The structures 'wash out' downstream because of the low resolution in that part the domain.

along the span of the cylinder. Each trace is characterized by its time-averaged value $\left(C_{L}\right.$ and $\left.C_{D}\right)$ and the root-mean-square variation of the instantaneous value around this average $\left(C_{L}^{\prime}\right.$ and $\left.C_{D}^{\prime}\right)$. The time-averaged lift coefficient is always zero. If the two-dimensional results were included in figure 20, the corresponding traces of $C_{L}(t)$ and $C_{D}(t)$ would both be perfectly time-periodic signals. Each three-dimensional calculation shows a varying degree of 'irregularity' that depends on the spanwise correlation of the flow. Because the signals are highly non-periodic it is difficult to 


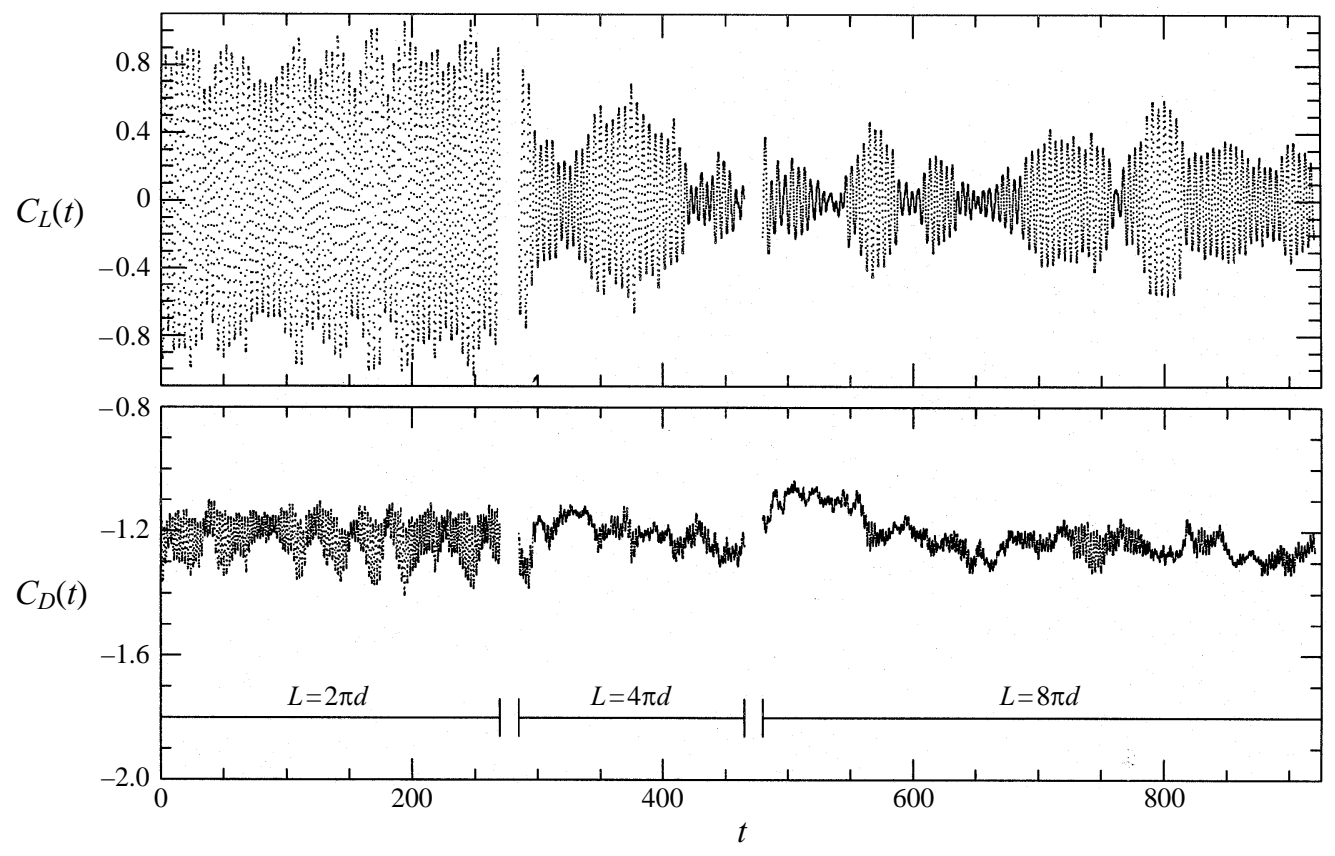

FiguRE 20. Unsteady lift and drag coefficients for the 'turbulent' flow past a cylinder at $R e=1000$. The data illustrate the effect increasing domain size has on the fluctuating forces, up to the maximum of $L=8 \pi d$. The $t$-axis is only for scale: short gaps between data sets are long transients in the computations.

estimate the asymptotic values of the fluctuating forces without taking averages over extremely long times, but the qualitative effect of increasing $L$ is quite clear.

In the smallest domain $(L=2 \pi d)$ the shedding is well-correlated along the span and the amplitude of the fluctuating lift and drag is quite large. The amplitude of the fluctuating component decreases with increasing $L$ due to the loss of spanwise correlation. On the largest domain $(L=8 \pi d)$ the force signals appear to be modulated, and there are times when the amplitude of the fluctuating lift falls almost to zero. Times of minimum $C_{L}^{\prime}(t)$ in figure 20 correspond to the appearance of phase dislocations in the wake. At these times the instantaneous force $\boldsymbol{F}(z, t)$ is misaligned due to the phase difference and there is a certain amount of cancellation when the force is averaged along the span. The time scale of the modulation is related to the rise and fall of energy in the low-wavenumber modes of the system. The modulation is not periodic like the beating that would occur with a simple nonlinear interaction between two temporal frequencies, for example between the vortex shedding frequency and the oscillation frequency of the separating shear layer. Instead the fluctuations appear in bursts at irregular intervals, consistent with their origin as a feature of the competition between low-wavenumber spatial modes in the wake.

Another interesting effect of large-scale structure in the wake can be seen in the comparison between instantaneous and time-averaged surface pressure distributions, shown in figure 21. This figure presents $C_{p}(\theta)$ at $R e=1000$ obtained from both experimental measurements and simulations of the three-dimensional flow. First note that the pressure distribution along the base of the cylinder is quite flat at this Reynolds number. This is also a direct result of weak spanwise correlation in a large system due to intrinsic three-dimensionality. The reduction in spanwise correlation 


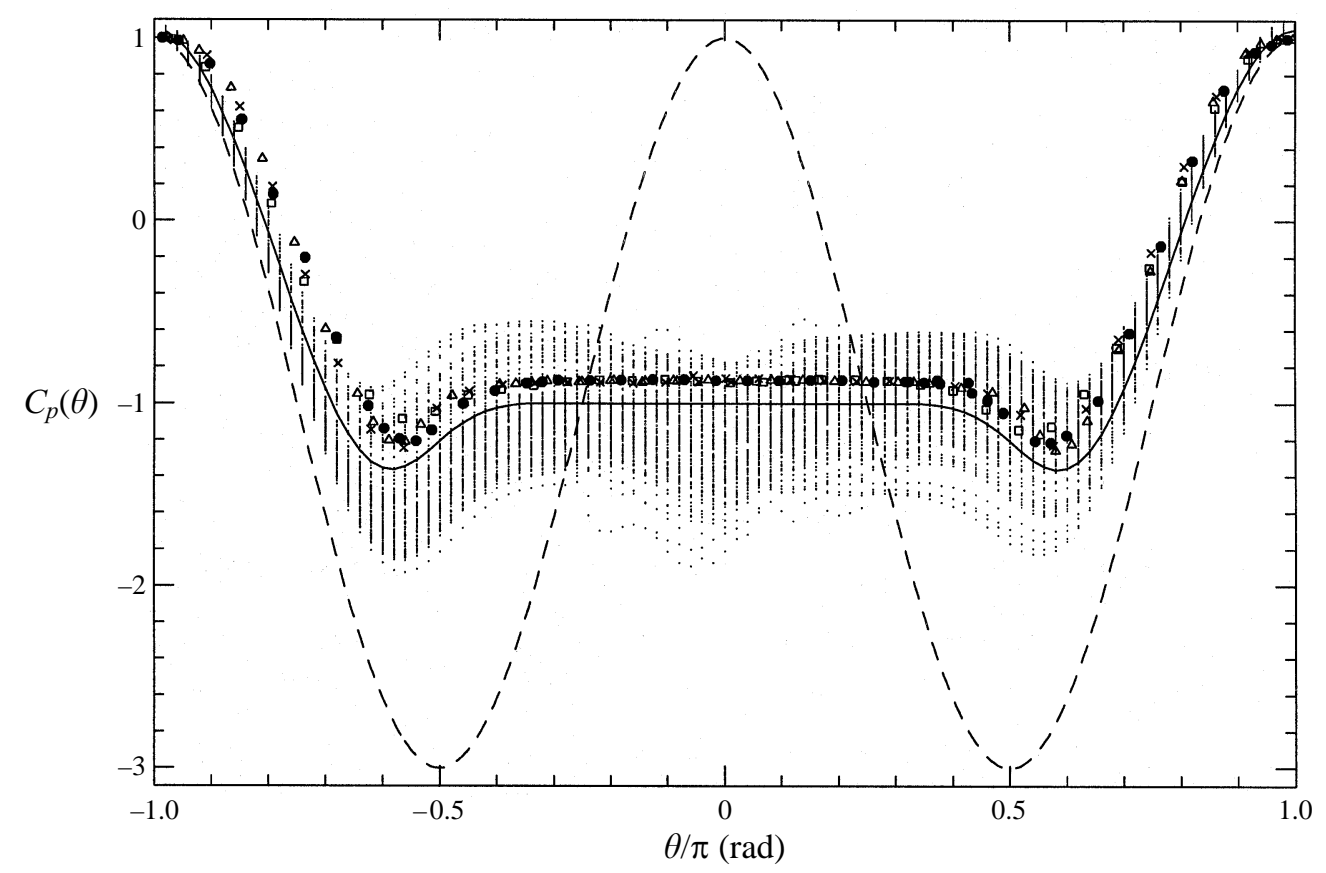

FiguRE 21. Surface pressure distribution for flow past a circular cylinder at $R e=1000$ : (symbols), experimental measurements by Leyva, Henderson \& Gharib (1996); the solid line is the time-averaged pressure distribution from a three-dimensional simulation with $L=8 \pi d$; the dashed line is the potential flow solution. The 'shaded' region indicates the instantaneous values of $C_{p}(\theta, z, t)$ at 64 locations along the span.

results in a smoothing of the mean pressure distribution. A strongly correlated flow would exhibit low-pressure peaks associated with the average position of vortex formation. For example, the surface pressure distribution at $R e=265$ shown in figure 17 exhibits these peaks because the three-dimensional flow remains wellcorrelated along the span. The 'shaded' region in figure 21 reveals further evidence of large phase differences by showing the instantaneous cross-sectional surface pressure coefficient $C_{p}(\theta, z, t)$ at 64 locations along the span. If the flow were well-correlated, this region would collapse into a narrow band (for the perfectly correlated twodimensional flow it is a single line). Since the solid line indicates the mean value of $C_{p}(\theta)$, and the shaded area extends equally far above and below the mean value in the base region, we can infer that at this instant there is a complete variation in phase along the span of the cylinder.

When plotted as in figure 21 the difference between the computations and experimental measurements for the surface pressure coefficient does not seem that large. In the simulated wake the value of $C_{p}$ at $\theta=0$ (the base pressure) is actually about $10 \%$ more negative. This difference also accounts for the discrepancy in the computed drag coefficient shown in figure 4. Although the shape of the curve is in good agreement with experiments, including the flatness of the base pressure region, the surface pressure coefficients obtained from the computation are uniformly lower than the experimental measurements. This suggests that there is more dissipation within the boundary layer and near wake in the laboratory flow than in the simulated flow, which could arise from any number of sources: free-stream turbulence interacting with the boundary layer, surface roughness, and so forth. It may also be due to a ne- 
glect of small-scale three-dimensionality in the computational model. So far attempts to isolate the discrepancy and close the gap between computation and experiment at this Reynolds number have been unsuccessful.

\section{Discussion}

At the present time there is still a vigorous debate over the precise physical origin of the wake instability. Researchers have sought a more physical explanation of the two-dimensional vortex shedding instability for almost a century now, so imagine the difficulty of describing the more complicated three-dimensional instability in simple physical terms! Instead we focus primarily on how instability modes in the wake determine the overall structure of the three-dimensional flow and the transition to 'turbulence' with increasing Reynolds number. The present study provides important clarification of various properties of modes $\mathrm{A}$ and $\mathrm{B}$ and their interaction in the transition range of $R e \approx 150$ to 300 . The calculations show good agreement with experimental observations of natural transition in the wake and highlight the role of the long-wavelength mode A instability in producing irregular dynamics at Reynolds numbers just beyond the onset of the secondary instability. In general the computations show that large-scale structure in the wake plays a vital role in the overall dynamics, not only near the onset of the secondary instability but also at higher Reynolds numbers as well.

\subsection{Nonlinear aspects of modes $A$ and $B$}

We begin by summarizing the properties of modes A and B that have been determined from stability calculations and direct numerical simulations, and how these properties compare with experimental observations. First, it is important to note that each instability is associated with a continuous wavenumber band, i.e. for $R e>R e_{2}$ there is not a single A mode, there is a branch of A modes. All modes along the A and $\mathrm{B}$ branches are formally related by a distinct spatiotemporal symmetry. Barkley \& Henderson (1996) report the complete form of these symmetries, both of which have been verified in experiments by Brede et al. (1996) and Williamson (1996b). The actual form is not so important for the present discussion so much as the fact that they are different. As a general rule, nonlinearity or some other pattern selection mechanism selects a single wavenumber from the continuous band of symmetry-related modes associated with the linear instability. Generally this mode would be observed in the physical system as a spatially periodic pattern centred around the critical wavelength $\lambda_{c}=2 \pi / \beta_{c}$. Since this is not consistent with experimental observations of threedimensionality in the wake of long circular cylinders, we do not assume that for $R e>R e_{2}$ there is a sharply defined mode A or mode $\mathrm{B}$. However, a pure bifurcation is easily arranged in the computations by selecting precise initial conditions and this is the case we consider first.

From linear and nonlinear stability calculations, mode $\mathrm{A}$ is found to appear as a subcritical bifurcation from the two-dimensional flow near $R e_{2} \simeq 190$ with a wavelength of $\lambda_{2}=3.96 d$ at onset. Owing to the subcritical nature of mode $\mathrm{A}$, the wake is also unstable to finite-amplitude perturbations at lower Reynolds number and there is a hysteresis in the transition between two-dimensional and three-dimensional states. In the ideal system this hysteresis occurs only in a small neighbourhood $R e=(1+\epsilon) R e_{2}$ of the critical point with a lower bound of $R e \approx 180$. Mode A is strongly coupled to the two-dimensional wake over a large distance downstream of the cylinder, producing a large-amplitude distortion of the Kármán vortices and a 
jump in forces acting on the cylinder. Bifurcation to pure mode A results in a threedimensional flow that is perfectly periodic in space and time, but with a significant drop in shedding frequency due to the strong nonlinear coupling between the primary and secondary instabilities.

Experimental measurements of the wavelength and transition Reynolds number for mode $\mathrm{A}$, including hysteresis near the critical point $R e_{2}$, show good agreement with linear and nonlinear stability calculations (Barkley \& Henderson 1996; Williamson 1996a). Figure 3 shows there is also good agreement between the computed and measured frequency drop near $R e_{2}$. Although there are important differences between the pure mode A state and experimental observations for $R e>R e_{2}$ (see \$4.3), the excellent agreement in these global quantities indicates they are determined primarily by the interaction between the primary instability and a single mode A instability. In sharp contrast, all previous numerical studies report a soft onset of three-dimensionality in the wake with a continuous variation of global quantities like shedding frequency and drag (Karniadakis \& Triantafyllou 1989; Tomboulides, Triantafyllou \& Karniadakis 1992; Noack \& Eckelmann 1994; Zhang et al. 1995). Previous discrepancies between simulation and experiment can be attributed to an unfortunate combination of domain size and resolution effects.

The present study also clarifies an important misconception regarding the change in shedding frequency at $R e_{2}$. Both Zhang et al. (1995) and Williamson $(1996 a-c)$ have speculated that this change only occurs in the presence of some other complicating phenomenon, and that 'pure mode A' corresponds to a higher frequency curve. In particular, speculation by Williamson $(1996 a-c)$ regarding two separate frequency curves A and $\mathrm{A}^{*}$ is largely incorrect. It is important to note that Williamson's statements were not based on direct measurements but on assumptions about the correlation between hot-wire data and flow visualization in separate facilities. Nonlinear calculations for the onset of mode A presented in $\$ 3.3$ clearly show that the shift in oscillation frequency is due to the coupling between mode A and the primary instability. The distinction between pure mode A and more complex flows is not the drop in magnitude but whether the frequency spectrum is broad-band or contains a single peak. The fact that frequency spectra measured by Williamson (1988) and Leweke \& Provansal (1995) are broad-band just above $R e_{2}$ indicates there is not a bifurcation to pure mode $\mathrm{A}$ in experiment that is stable at long times.

Calculations at higher Reynolds number show that mode $\mathrm{B}$ appears as a supercritical bifurcation from the two-dimensional flow at $R e_{2}^{\prime} \simeq 260$ with a wavelength of $\lambda_{2}^{\prime}=0.822 d$ at onset. Unlike mode A, this instability occurs over a narrow range of wavelengths and the transition is sharp and without hysteresis. Mode B is primarily a near-wake instability that interacts weakly with the Kármán vortex street and decays rapidly with distance downstream of the cylinder. Near the critical point there is no direct change in oscillation frequency. In fact, because of the supercritical nature of the instability, there is a continuous bifurcation from the two-dimensional branch to pure mode $\mathrm{B}$.

Previous numerical studies have not examined the precise onset of mode B as a bifurcation from the two-dimensional wake, and the supercritical bifurcation cannot be verified directly by experiment since this instability occurs in a range of Reynolds number where the wake is already unstable to mode A. However, experiments do indicate a sharp and non-hysteretic change at $R e_{2}^{\prime} \simeq 260$ in measurable quantities like shedding frequency, base pressure coefficient, and drag. Prasad \& Williamson (1996) and Williamson (1996b) speculate on a possible 'resonance' between the vortex shedding frequency and a two-dimensional shear layer instability of the type observed 
at $R e>1000$. However, the shear layer seems entirely stable to two-dimensional perturbations at low Reynolds number and there is no evidence of any such resonance in the present study. The changes in wake response at $R e_{2}^{\prime} \simeq 260$ seem related solely to the nonlinear stability of mode $\mathrm{B}$ and provide indirect experimental evidence that mode $B$ is supercritical.

One of the open questions is why mode $\mathrm{B}$ is observed at $R e<R e_{2}^{\prime}$. Numerous experimental studies report periodic disturbances with a spanwise wavelength of around one diameter for Reynolds number as low as $R e \approx 200$. Similar structures are reported in computational studies by Zhang et al. (1995) at $R e=220$, Thompson et al. (1996) at $R e=250$, and in the present study at $R e=220$. There are three important facts to consider in this regard: (i) modes A and B are unrelated by symmetry and only coupled indirectly through the changes they induce in the primary instability mode; (ii) mode $\mathrm{B}$ only appears at $R e<R e_{2}^{\prime}$ in the presence of mode A; and (iii) a small amount of mode B leads to a large reduction in the amplitude of mode A. The present study provides preliminary validation for a theory and nonlinear model proposed by D. Barkley (private communication, 1996) that combines each of these facts. In short, the two modes interact only through the changes they induce in the primary instability, and that interaction is such that mode A de-stabilizes mode B while mode B re-stabilizes mode A. Flow visualization and frequency calculations presented in $\$ 3.5$ show these effects directly. A nonlinear coupling of this form would explain why mode A is difficult to observe at higher Reynolds number and why the shedding frequency returns close to its two-dimensional value near $\operatorname{Re}_{2}^{\prime}$. Additional calculations confirming this scenario and identifying the precise Reynolds number where mode A (rather than the two-dimensional flow) first becomes unstable to mode B are the subject of current work.

We close this section with a final question regarding the general nature of global modes in the wake, namely: Do the global modes (eigenfunctions of the temporal stability equations) provide the true three-dimensional structure of the flow after transition? In general this depends on how 'nonlinear' the final state is. Because the bifurcation to mode A is strongly subcritical, the nonlinear state after mode A saturates is significantly different from the linear superposition of the leading eigenmode and the two-dimensional flow. This can be seen by comparing figure 7 in the present study with figure 9 of Barkley \& Henderson (1996). The global mode only describes the three-dimensional structure of the flow accurately when $|A| \ll\left|U_{0}\right|$. On the other hand, the bifurcation to mode B is supercritical and therefore saturates at low amplitude before nonlinearity distorts the structure of the bifurcating global mode. In both cases the global mode determines the wavelength and spatiotemporal symmetry after transition, but strong nonlinearity will always distort the mode shape.

\subsection{Origin of large-scale structure in the wake}

Another interesting feature of transition in the wake is the observation of threedimensional structure with a spanwise scale much larger than the secondary instability wavelength. Williamson (1992) referred to such structures as spot-like 'vortex dislocations' and indicated they could grow to a size of the order 10 to 20 primary instability wavelengths downstream of the cylinder (a spanwise wavelength of the order 50 to 100 diameters). They are the essential feature of the state he referred to as $\mathrm{A}^{*}$, meaning mode A plus 'vortex dislocations'. The term dislocation in this context is somewhat misleading since it usually refers to a highly localized disturbance in an otherwise ideal pattern, whereas a 'vortex dislocation' is a decidedly non-local phenomenon that fills a large region of space. For example, compare the 'vortex 
dislocations' in figure 13 and the 'phase dislocations' in figure 19. Presumably viscous effects prevent the formation of true pattern dislocations at low Reynolds number. Zhang et al. (1995) observed a related type of three-dimensional shedding near $R e_{2}$ which they referred to as a 'vortex adhesion' mode. Because of the strong similarity between these structures and the development of turbulent spots in boundary layer transition (see Williamson 1992), they will be referred to here simply as 'spot-like' disturbances rather than dislocations.

There is probably a wide variety of interesting patterns that can be produced by exciting various nonlinear responses in the wake. Williamson (1992) produced 'vortex dislocations' artificially with a small ring mounted on the surface of the cylinder. This type of sharp local disturbance along the span is equivalent to a near-wake perturbation of three-dimensional global modes across a broad band of wavenumbers - the sharper the disturbance in physical space, the broader the perturbation in wavenumber space. Zhang et al. (1995) produced 'vortex adhesion' in their simulations of the wake by inserting a "strong localized spanwise inhomogeneity in the initial conditions," which is the same type of effect. In their calculations "vortex adhesion' did not arise naturally from small perturbations. Spot-like disturbances in the present study always develop from small perturbations for sufficiently large $R e>R e_{2}$ and $L>\lambda_{2}$, and they occur specifically during the rapid nonlinear growth of subdominant mode A instabilities.

Although each study cited above (including the present one) visualizes somewhat different quantities, there are certain characteristics that identify these as essentially the same phenomena. Large-scale structures only appear in natural transition after mode A, i.e. after the fastest-growing instability mode saturates. Like the global instabilities that drive all three-dimensionality in the wake, these disturbances develop simultaneously over large distances downstream of the cylinder. They are initiated outside the near wake and slightly downstream of the cylinder where the local amplitude of mode $\mathrm{A}$ is large. They have an overall $\Lambda$-shaped appearance that may point either upstream or downstream (see figure 13), and the core of the disturbance is aligned at a given spanwise location for a significant distance downstream. Figure 14 showed that a vortical structure indicative of mode A lies at the core of these disturbances.

In the absence of external forcing (e.g. end effects, imperfections in the geometry) or phenomena induced by the initial conditions, all large-scale structure must be generated by the mode A instability. There is no evidence of other instabilities that would energize and sustain such large-scale disturbances. In $\$ 3.5$, spot-like disturbances in the wake developed naturally during the breakdown of the ideal spatially periodic mode A pattern due to the interaction with subdominant mode A instabilities. This process does not depend on any non-uniformity in the surface of the cylinder or the approaching free-stream flow, nor does it depend on end effects. It only depends on the broad-band nature of the mode A instability and the lack of a strong pattern selection mechanism to lock the system onto a single three-dimensional global mode and suppress other subdominant but self-excited modes of the wake. Williamson (1996b) interprets this phenomenon as "a local spanwise location where the shedding frequency falls out-of-phase with the shedding to each of the two sides," i.e. a phase dislocation. The present study indicates these disturbances are part of the intrinsic three-dimensional structure of the wake and not simply a local variation in the phase of vortex shedding. Unlike experiment, spot-like disturbances in the simulations represent a transient phenomenon that only occurs during the breakdown of the spatially periodic flow and the evolution towards a long-time energy spectrum 
of the form shown in figure 16. Their persistence in experiment may be related to end effects. However, it is clear that their initial appearance results from the growth of subdominant instability modes and represents another important nonlinear aspect of the mode A instability.

\subsection{Irregular dynamics and fast transition to 'turbulence'}

We begin this section with a summary of the most important experimental observations related to transition in the wake of large-aspect-ratio systems. For a more comprehensive discussion see the recent review by Williamson (1996c). The following comments are based primarily on experimental results presented by Williamson (1988, 1991, 1992, 1996a-c), Mansy, Yang \& Williams (1994), Leweke \& Provansal (1995), Zhang et al. (1995), Wu et al. (1996), and Brede et al. (1996). There is generally good agreement that the transition from two-dimensional vortex shedding to a threedimensional state occurs at $R e_{2} \simeq 190$, and that the transition shows a hysteresis with respect to Reynolds number. Velocity fluctuations in the wake exhibit broad-band frequency spectra just beyond the onset of this instability and a significant drop in shedding frequency from that of the two-dimensional flow. Williamson $(1996 a-c)$ shows that broadening of the spectrum is not due to slow variations in shedding phase or random fluctuations, but is directly associated with the appearance of 'vortex dislocations' in the wake. Regular mode A vortex shedding is only observed as a transient in the approximate range $R e \approx 180$ to 200 , and experimental wavelength measurements show considerable scatter. At long times the flow is highly irregular, reinforcing the fact that there does not appear to be a bifurcation to pure mode A in experiment that is stable at long times. In contrast to this, measurements for the wavelength of mode $\mathrm{B}$ fall into a narrow band with good agreement among various experimental groups. Mode $\mathrm{B}$ is observed from $R e \approx 200$ on, and as $R e$ approaches $R e_{2}^{\prime} \simeq 260$ there is a reasonably well-defined wavelength in the near wake and a sharp peak in the frequency spectrum. However, this peak is superimposed over a broad band of frequencies in the background indicative of 'turbulence' in the wake farther downstream.

In comparing computational and experimental results for transition it is first important to make a more precise distinction between aspect ratio and spanwise dimension. In computation, a moderate value of $L$ corresponds to a system which is restricted to a small number of discrete modes, even though the system is infinitely large (spatially periodic). For example, the calculations presented in $\$ 3.3$ represent the interaction between the primary instability $(\beta=0)$ and the critical mode A instability $\left(\beta=2 \pi / \lambda_{2}\right)$ in an infinite domain. Longer-wavelength modes are excluded by restricting the dynamics to a subspace with spanwise dimension $L=\lambda_{2}$. An aspect ratio much larger than $L=\lambda_{2}$ is needed in experiment so this interaction is not polluted by end effects, and therefore experiments with small aspect ratio are in no way similar to computations with moderate $L$. The lack of end effects in computation makes it possible to classify the response of the flow to specific perturbations and allows a more systematic study of phenomena associated with modes A and B.

Transition results from the present study are summarized in table 2. Unlike experimental systems, perturbations near either critical point converge to a threedimensional, time-periodic flow (a limit cycle). However, time-periodic flows are only observed in systems with a small spanwise dimension $\left(L=\lambda_{2}\right.$ or $\left.L=\lambda_{2}^{\prime}\right)$ where the dynamics represent a single global oscillator. Increasing spanwise dimension $L$ leads to spatiotemporal chaos driven by the competition between multiple self-excited mode $\mathrm{A}$ instabilities. In these cases the spatial pattern associated with pure mode $\mathrm{A}$ 


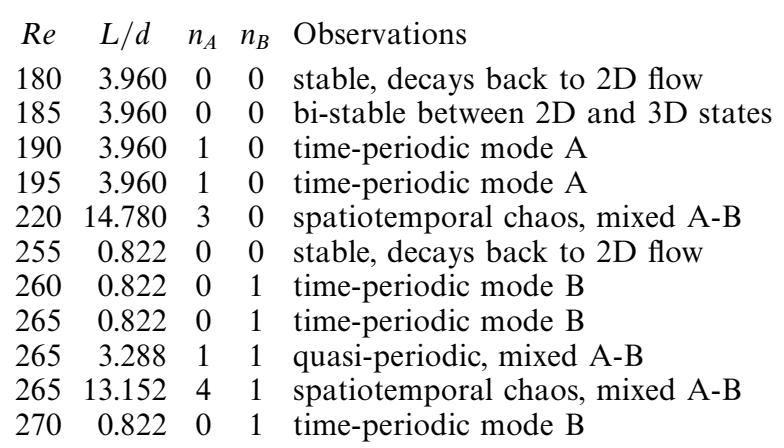

TABLE 2. Summary of observed final states as a function of $R e$ and $L$ for calculations in the transition range, $R e=150$ to 300 . Parameters $n_{A}$ and $n_{B}$ indicate the number of discrete wavenumbers in the system that fall into a region of instability in figure 1 . Note that chaotic flows are only observed for $n_{A} \geqslant 3$ over this range of Reynolds number.

occurs only as a transient, and turbulent structures like those observed in experiment appear just prior to the breakdown of the spatially periodic mode A pattern and subsequent transition to a chaotic state. Mode B is only observed in large systems as a short-wavelength pattern superimposed over a background of large-scale irregular motions. For example, compare the regularity of the near wake and the irregular flow slightly downstream in figures 13 and 14. With increasing Reynolds number the excitation due to mode $\mathrm{A}$ is spread across a wider band of wavenumbers and any coherent mode A pattern disappears. Mode B can be clearly identified over a wider range of Reynolds number because it is limited to a relatively narrow range of wavelengths. However, the computed spanwise energy spectrum (figure 16) indicates that mode $\mathrm{A}$ is the primary source of excitation in the three-dimensional wake.

We can now outline the properties of the system that would lead one to expect chaotic behaviour and identify a simple mechanism for the onset of irregular dynamics and fast transition to turbulence in the wake. Spatiotemporal chaos is a common feature of systems where excitation occurs at a length scale much smaller than the system size but larger than the dissipation scale $\left(L \gg l_{E}>l_{D}\right)$. Mode A instability in the wake fits this scenario well. The excitation scale $l_{E} \approx \lambda_{2}$ is fixed by the finitewavenumber instability of mode A, which is in turn fixed by the length scale of the primary instability (spacing of the Kármán vortices). The subcritical nature of the bifurcation to mode A shows that $l_{E}>l_{D}$ at onset. When $L \approx l_{E}$ the dynamics are regular or quasi-periodic $\left(\$ \S 3.3,3.4\right.$, and 3.5.1), but when $L \gg l_{E}$ the flow exhibits spatiotemporal chaos ( $\$ 3.5 .2$ and 3.6). Calculations at $R e=265$ established that chaotic states first arise from the competition between multiple mode A instabilities. For any $R e>R e_{2}$ there is a minimum spanwise dimension $L$ such that three or more discrete modes lie within the mode A instability region. This can be estimated from the width of the instability region in figure 1 and was used to determine appropriately large spanwise dimensions at $R e=220$ and $R e=265$. The absolute (self-excited) nature of the wake instability guarantees that each of these modes will grow, setting up a competition between multiple oscillators represented by the global modes. The dynamics in larger systems are necessarily chaotic because we can always choose initial conditions that lie in the same (chaotic) subspace. We conclude that the wake follows the Ruelle-Takens-Newhouse (RTN) route to turbulence for fixed $R e>R e_{2}$ 
and $L \rightarrow \infty$. This a universal route to turbulence in dissipative systems that develop three or more incommensurate frequencies (Ruelle \& Takens 1971; Newhouse, Ruelle $\&$ Takens 1978). As Reynolds number is increased beyond $R e_{2}$ the flow gradually enters the regime of strong turbulence due to the increasing separation in scale between excitation due to the secondary instability (fixed length scale, $l_{E} \approx \lambda_{2}$ ) and dissipation due to viscosity (decreasing length scale, $l_{D} \sim R e^{-1 / 2}$ ).

At least two other scenarios have been proposed for the 'route to turbulence' in the wake. The onset of chaotic dynamics agrees qualitatively with the onset of spatiotemporal chaos due to the Benjamin-Feir instability in the complex GinzbergLandau (GL) model. Leweke \& Provansal $(1994,1995)$ proposed a certain variation of parameters in this equation that correctly models the change in oscillation frequency during transition and mimics the complex dynamics of the wake near the onset of the secondary instability. However, the GL model does not have a finite-wavenumber instability and therefore cannot quantitatively describe the secondary instability of the wake due to mode A. An extension of the discrete model used in the present study to the case of a coupled set of instability modes may lead to a more complete model for wake transition, but this requires a fundamentally different approach from studying instabilities in the GL model.

In simulations of the three-dimensional wake, Karniadakis \& Triantafyllou (1992) and Tomboulides et al. (1992) observed a period-doubling bifurcation at $R e \approx 300$ and proposed that the wake might follow a period-doubling route to turbulence. Perioddoubling occurred for fixed system size $L \approx 1.57 d$ and increasing control parameter $R e \rightarrow \infty$. The small size of their system excluded the mode A instability altogether. Mittal \& Balachandar (1995b) also report a mechanism related to period-doubling in the wake at $R e=500$, but their calculations were for an equally small system of size $L \approx 1 d$. A period-doubling cascade seems reasonable when the complex dynamics of the large system are eliminated by such severe restrictions on the spanwise dimension L. Recent experiments by Williams, Mansy \& Abouel-Fotouh (1996) show evidence of subharmonic fluctuations and a shifting of the three-dimensional structure of the flow along the span of the cylinder at $R e=300$, so there may be some elements of the physical mechanism suggested by Tomboulides et al. (1992) related to the dynamics of the near wake. However, in the light of the overwhelming experimental data in the transition range and computational results presented here for large systems, it is clear that the wake does not follow a period-doubling route to turbulence in any rigorous sense.

We are still left with the following question: Does the irregular behaviour reported here and observed in experiment represent deterministic chaos or stochastic chaos? A precise answer to this question in terms of Lyapunov exponents or generalized attractor dimensions can be extremely difficult to quantify for large systems. This is an important characterization of the wake dynamics, but at the present time the computational expense of this type of analysis is prohibitive. However, there is good evidence that the simulations reported here exhibit deterministic chaos. First, we saw that perturbations to a single instability mode produce a time-periodic (regular) state. For fixed Reynolds number and equivalent numerical resolution, chaotic behaviour is only observed when three or more incommensurate mode A instabilities are excited. Since stochastic forcing in these calculations arises from machine errors (e.g. finiteprecision arithmetic), the evolution of the spanwise energy spectrum relative to the level of machine errors is a critical comparison. Figure 22 shows this for the complete calculation from small perturbation to spatiotemporal chaos at $R e=265$ in the system with $L=13.152 d$. Clearly the dynamics are regular when the amplitude 


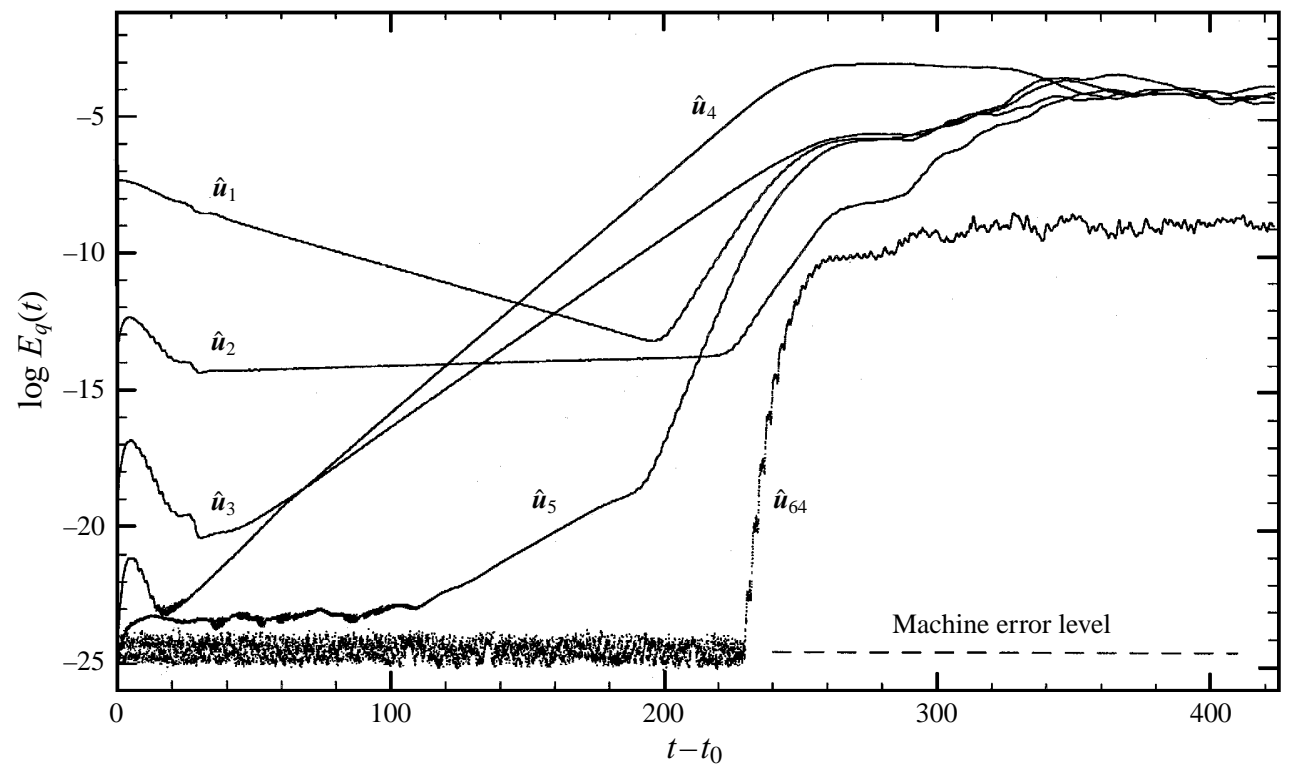

FigURE 22. Time-dependent energy spectrum for the complete calculation presented in figure 15 . Traces show the evolution of the linearly stable long-wavelength mode $\left(\hat{\boldsymbol{u}}_{1}\right)$, the four fundamental instability modes $\left(\hat{\boldsymbol{u}}_{2-5}\right)$, and the highest harmonic retained in the calculation $\left(\hat{\boldsymbol{u}}_{64}\right)$. The latter exhibits stochastic forcing due to machine errors at early times, but the irregular behaviour of the system at long times is due solely to the nonlinear competition between self-excited modes of the wake.

of each instability mode is small but growing exponentially (the linear and weakly nonlinear regimes). Irregular behaviour arises from the strong nonlinear interaction between these modes that only occurs at later times when they reach a sufficiently large amplitude. In the asymptotic state there is a separation of twenty orders of magnitude between the excitation level due to the wake instability and stochastic forcing due to machine errors. This does not constitute proof of deterministic chaos, but given the link with the RTN mechanism of competing modes, 'deterministic chaos' is a reasonable conclusion.

\subsection{Effect of large scales on fluctuating forces}

Experimentally it is observed that the fluctuating lift and drag on bluff bodies at higher Reynolds number is not strictly periodic but appears in bursts. The bursting intervals are much longer than the primary shedding frequency. Similar behaviour is reported for flow past circular cylinders by Szepessy \& Bearman (1992) and for bluff plates by Lisoski (1993). Szepessy \& Bearman associate this behaviour with the spanwise modulation of the vortex shedding phase. Both studies note that the time scale of the modulation depends on the aspect ratio of the system, $L / d$. Therefore, it has not been clear whether the modulation of forces is due somehow to end effects or to intrinsic three-dimensionality in the flow.

In the present study the modulation of forces is found to originate in the intrinsic large-scale structure of the wake. In previous computations, Mittal \& Balachandar $(1995 a)$ show that three-dimensionality on a small scale $(\lambda \approx 1 d)$ modifies the Reynolds stresses in the near wake, and this is the primary factor in reducing the mean drag. The main effect of large scales $(\lambda \gg 1 d)$ seems to be the modulation of fluctuating lift and drag through changes in the local phase of vortex shedding. Figure 20 shows 
that this modulation is absent in small systems where the flow is roughly correlated along the span. As we increase the system size $L$, spanwise correlation is lost and the flow exhibits large-amplitude variations in the phase of vortex shedding. Figure 19 shows this occurring in the extreme case of phase dislocations along the span of the cylinder. The shedding frequency is constant within a 'cell' that may be in phase or out of phase with respect to adjacent cells. Over long times the variation in phase produces a relatively flat pressure distribution along the base of the cylinder, as shown in figure 21. This is clearly not possible in a small spanwise-correlated system, and may be taken as direct evidence that the large-scale structure of the wake is important at higher Reynolds number.

\subsection{Concluding remarks}

The flow past a circular cylinder exhibits spatiotemporal chaos at Reynolds numbers just beyond the onset of the secondary instability. Irregular dynamics and fast transition to 'turbulent' flow are due primarily to the broad-band nature of the mode A instability in the wake and the competition between self-excited global modes that arise from this instability. This was shown directly from computations at $R e=220$ and 265, and is consistent with measured frequency spectra and experimental flow visualization at lower Reynolds numbers. Regular flow patterns associated with both mode A and mode B occur either as transients or when the flow dynamics are severely restricted by imposing spanwise periodicity. The focus on spatial energy spectra and large-scale flow patterns in the present study clarifies the relative importance of these instabilities at higher Reynolds number, and indicate that at $R e>R e_{2}$ mode $\mathrm{A}$ is the dominant source of energy transfer from the primary instability mode (the Kármán vortex street) to the turbulent, three-dimensional flow. Future computational studies that address turbulence in the wake by either direct or large-eddy simulation should include scales of at least $l_{E} \approx \lambda_{2}$, preferably an order of magnitude larger. Mode $\mathrm{B}$ develops as a second, separate instability process that primarily affects the near wake. Since these modes have different symmetries and occur on different length scales, there is not a direct transition or transfer of energy from one mode to the other. Nonlinear interaction between self-excited modes in the A-band is responsible for the appearance of large-scale structures in the wake during the breakdown of the periodic three-dimensional state. Simulations at higher Reynolds number show that the flow can develop localized phase dislocations and that large-scale structure in the wake is responsible for modulating the amplitude of fluctuating lift and drag. There is no direct evidence in the present study of separate three-dimensional instabilities or phenomena that cannot be explained in terms of the mode A and mode B instabilities.

Questions regarding the nature of the turbulent wake at large Reynolds number must ultimately address the evolution of the flow over long times and large distances. Such calculations are particularly demanding in terms of computational resources, and it is difficult to provide a reliable analysis of asymptotic behaviour in open flow systems based on brute-force integration of the Navier-Stokes equations. In the present study the three-dimensional flow at moderate Reynolds number is represented by a dynamical system with about one million degrees of freedom. It takes approximately $5000 \mathrm{~s}$ to simulate one shedding cycle at this resolution using the available computer resources. The time scale for a typical experiment in air at $R e \approx 1000$ is about $0.005 \mathrm{~s}$ per shedding cycle, giving a ratio of time scales between experiment and computation of the order one million to one. This comparison is made simply to point out the challenge facing realistic DNS of wake turbulence and to motivate the need for appropriate model systems. 
On a more positive note, the flow past a circular cylinder is an ideal problem for computation because the entire sequence of states from steady flow to 'turbulence' can be studied in an extremely small range of Reynolds number. As Roshko (1954) first suggested, the transition to 'turbulence' is essentially complete at $R e \approx 300$. Within this small parameter range there is a supercritical Hopf bifurcation, subcritical and supercritical pitchfork bifurcations, coherent structures, complex vortex dynamics, and spatiotemporal chaos ... all for the simplest case of uniform flow past an infinitely long cylinder! Even though the circular cylinder has served as the most important prototype bluff body flow for almost a century, the quantitative description of these phenomena has only begun to unfold in the past few years. As H. W. Liepmann is so fond of saying, one can always discover interesting new things in the most classical problems.

This work would not have been possible without the assistance of Dwight Barkley at the University of Warwick - his help is greatly appreciated! The author would also like to acknowledge several inspiring discussions with Michael Cross at Caltech, as well as questions and critical feedback from a number of other people: H. Blackburn, M. Gharib, A. Leonard, D. Hill, D. Meiron, A. Roshko, C. H. K. Williamson, S. Balachandar, H.-Q. Zhang, P. Monkewitz and the referees. Financial support was provided by the NSF through Grant No. CDA-9318145 and the ONR through Grant No. N000-94-1-0793. Computational resources were provided by the Center for Advanced Computing Research and the JPL High Performance Computing and Communications program at the California Institute of Technology.

\section{REFERENCES}

BARKLey, D. \& Henderson, R. D. 1996 Three-dimensional Floquet stability analysis of the wake of a circular cylinder. J. Fluid Mech. 322, 215-241.

Bernard, C., Maday, Y. \& Patera, A. T. 1992 A new nonconforming approach to domain decomposition: the mortar element method. In Nonlinear Partial Differential Equations and their Applications (ed. H. Brezis \& J. L. Lims). Pitman and Wiley.

Blackburn, H. M. \& Melbourne, W. H. 1996 The effect of free-stream turbulence on sectional lift forces on a circular cylinder. J. Fluid Mech. 306, 267-292.

Brede, M., EcKelmann, H. \& Rockwell, D. 1996 On secondary vortices in the cylinder wake. Phys. Fluids 8, 2117-2124.

Browand, F. K. \& Prost-Domasky, S. A. 1990 Experiment on pattern evolution in the 2-D mixing layer. In New Trends in Nonlinear Dynamics and Pattern Forming Phenomena (ed. P. Coullet \& P. Huerre), p. 159. NATO ASI Series 8, Plenum.

Browand, F. K. \& Troutt, T. R. 1980 A note on the spanwise structure in the two-dimensional mixing layer. J. Fluid Mech. 97, 771-781.

BRowand, F. K. \& TroutT, T. R. 1985 The turbulent mixing layer: geometry of large vortices. $J$. Fluid Mech. 158, 489-509.

Cross, M. C. \& Hohenberg, P. C. 1993 Pattern formation outside of equilibrium. Rev. Mod. Phys. 65, 851-1112.

Gerrard, J. H. 1978 The wakes of cylindrical bluff bodies at low Reynolds number. Phil. Trans. R. Soc. Lond. 288, 351-382.

Hama, F. R. 1957 Three-dimensional vortex pattern behind a circular cylinder. J. Aeronaut. Sci. 24, 156.

Hammache, M. \& Gharib, M. 1991 An experimental study of the parallel and oblique vortex shedding from circular cylinders. J. Fluid Mech. 232, 567-590.

HENDERSON, R. D. 1994 Unstructured spectral element methods: parallel algorithms and simulations. $\mathrm{PhD}$ thesis, Princeton University.

Henderson, R. D. 1995 Details of the drag curve near the onset of vortex shedding. Phys. Fluids 7, 2102-2104. 
Henderson, R. D. \& Barkley, D. 1996 Secondary instability in the wake of a circular cylinder. Phys. Fluids 8, 1683-1685.

Henderson, R. D. \& Karniadakis, G. E. 1995 Unstructured spectral element methods for simulation of turbulent flows. J. Comput. Phys. 122, 191-217.

Hohenberg, P. C. \& Shraiman, B. I. 1989 Chaotic behavior of an extended system. Physica D 37, $109-115$.

Huerre, P. \& Monkewitz, P. A. 1990 Local and global instabilities in spatially developing flows. Ann. Rev. Fluid Mech. 22, 473-537.

JACKSON, C. P. 1987 A finite-element study of the onset of vortex shedding in flow past variously shaped bodies. J. Fluid Mech. 182, 23-45.

Karniadakis, G. E., Israeli, M. \& Orszag, S. A. 1991 High-order splitting methods for the incompressible Navier-Stokes equations. J. Comput. Phys. 97, 414-443.

Karniadakis, G. E. \& Orszag, S. A. 1993 Nodes, modes, and flow codes. Phys. Today 46, 34-42.

Karniadakis, G. E. \& Triantafyllou, G. S. 1989 Frequency selection and asymptotic states in laminar wakes. J. Fluid Mech. 199, 441-469.

Karniadakis, G. E. \& Triantafyllou, G. S. 1992 Three-dimensional dynamics and transition to turbulence in the wake of bluff objects. J. Fluid Mech. 238, 1-30.

Leweke, T. \& Provansal, M. 1994 Model for the transition in bluff-body wakes. Phys. Rev. Lett. 72, 3174-3177.

Leweke, T. \& Provansal, M. 1995 The flow behind rings - bluff-body wakes without end effects. J. Fluid Mech. 288, 265-310.

Leyva, I., Henderson, R. D. \& Gharib, M. 1996 On the accuracy of pressure measurements for circular cylinders. GALCIT (unpublished).

Lisoski, D. 1993 Nominally 2-dimensional flow about a normal flat plate. PhD thesis, California Institute of Technology.

Mansy, H., YAng, P.-M. \& Williams, D. R. 1994 Quantitative measurements of three-dimensional structures in the wake of a circular cylinder. J. Fluid Mech. 270, 277-296.

Mathis, C., Provansal, M. \& Boyer, L. 1987 Bénard-von Kármán instability: Transient and forced regimes. J. Fluid Mech. 182, 1-22.

Meiburg, E. \& Lasheras, J. C. 1988 Experimental and numerical investigation of the threedimensional transition in plane wakes. J. Fluid Mech. 190, 1-37.

Mittal, R. \& Balachandar, S. $1995 a$ Effect of three-dimensionality on the lift and drag of nominaly two-dimensional cylinders. Phys. Fluids 7, 1841-1865.

Mittal, R. \& BalachandaR, S. $1995 b$ Generation of streamwise vortical structures in bluff body wakes. Phys. Rev. Lett. 75, 1300-1303.

Newhouse, S., Ruelle, D. \& Takens, F. 1978 Occurence of strange axiom A attractors near quasi periodic flows in $T^{m}, m \geqslant 3$. Commun. Math. Phys. 64, 35-40.

Noack, B. R. \& Eckelmann, H. 1994 A global stability analysis of the steady and periodic cylinder wake. J. Fluid Mech. 270, 297-330.

Patera, A. T. 1984 A spectral element method for Fluid Dynamics; Laminar flow in a channel expansion. J. Comput. Phys. 54, 468-488.

Prasad, A. \& Williamson, C. H. K. 1996 The instability of the separated shear-layer from a bluff-body. Phys. Fluids 8, 1347-1349.

Roshko, A. 1954 On the development of turbulent wakes from vortex streets. NACA Rep. 1191.

Ruelle, D. \& Takens, F. 1971 On the nature of turbulence. Commun. Math. Phys. 20, 167-192.

SZEPESSY, S. \& BEARMAN, P. W. 1992 Aspect ratio and end plate effects on vortex shedding from a circular cylinder. J. Fluid Mech. 234, 191-217.

Thompson, M., Hourigan, K. \& Sheridan, J. 1996 Three-dimensional instabilities in the wake of a circular cylinder. Exp. Therm. Fluid Sci. 12, 190-196.

Tomboulides, A. G., Triantafyllou, G. S. \& Karniadakis, G. E. 1992 A new mechanism of period doubling in free shear flows. Phys. Fluids A 4, 1329-1332.

Wieselsberger, C. 1921 Neuere Feststellungen über die Gesetze des Flüssigkeits- und Luftwiderstands. Phys. Z. 22, 321-238.

Williams, D. R., Mansy, H. \& Abouel-Fotouh, A. 1996 Three-dimensional subharmonic waves during transition in the near-wake region of a cylinder. Phys. Fluids 8, 1476-1485. 
Williamson, C. H. K. 1988 The existence of two stages in the transition to three dimensionality of a cylinder wake. Phys. Fluids 31, 3165-3168.

Williamson, C. H. K. 1989 Oblique and parallel modes of vortex shedding in the wake of a circular cylinder at low Reynolds numbers. J. Fluid Mech. 206, 579-627.

Williamson, C. H. K. 1991 Three-dimensional aspects and transition of the wake of a circular cylinder. In Turbulent Shear Flows 7, pp. 173-194. Springer.

Williamson, C. H. K. 1992 The natural and forced formation of spot-like 'vortex dislocations' in the transition of a wake. J. Fluid Mech. 243, 393-441.

Williamson, C. H. K. $1996 a$ Mode A secondary instability in wake transition. Phys. Fluids 8, $1680-1682$.

Williamson, C. H. K. $1996 b$ Three-dimensional wake transition. J. Fluid Mech. 328, 345-407.

Williamson, C. H. K. 1996 c Vortex dynamics in the cylinder wake. Ann. Rev. Fluid Mech. 28, 477-539.

Wu, J., Sheridan, J., Welsh, M. C. \& Hourigan, K. 1996 Three-dimensional vortex structures in a cylinder wake. J. Fluid Mech. 312, 201-222.

Zebib, A. 1987 Stability of viscous flow past a circular cylinder. J. Engng Maths 21, 157.

Zhang, H.-Q., Fey, U., Noack, B. R., König, M. \& Eckelmann, H. 1995 On the transition of the cylinder wake. Phys. Fluids 7, 779-794. 